\title{
Equilibrium thermodynamics of homopolymers and clusters: Molecular dynamics and Monte Carlo simulations of systems with square-well interactions
}

\author{
Yaoqi Zhou and Martin Karplus \\ Department of Chemistry and Chemical Biology, Harvard University, Cambridge, Massachusetts 02138
}

John M. Wichert and Carol K. Hall

Department of Chemical Engineering, North Carolina State University, Raleigh, North Carolina 27695-7905

(Received 19 May 1997; accepted 12 September 1997)

\begin{abstract}
The thermodynamics of homopolymers and clusters with square-well interactions of up to 64 particles are studied with constant-temperature discontinuous molecular dynamics (DMD) simulations; for comparison Monte Carlo (MC) simulations are also reported. Homopolymers composed of more than five beads are found to exhibit two or more equilibrium transitions. In the long chain limit, these multiple transitions correspond to gas-to-liquid, liquid-to-solid, and solid-to-solid transitions. In particular, the liquid-to-solid-like disorder-to-order transition for isolated 32mers and 64mers is strongly first order (bimodal energy distribution) at the reduced square-well diameter $\lambda=1.5$. As $\lambda$ decreases from 1.5 to 1.3 , the bimodal distribution becomes unimodal. The use of Lindemann's rule for solids indicates that the structure formed right below the liquid-to-solid transition temperature has a solid core but a liquid surface. Comparing the homopolymer results with those for square-well clusters indicates that the bonding constraint in homopolymers increases the temperatures of transitions but decreases their strength. The solid structure of an isolated $64 \mathrm{mer}$ is nearly identical to that of a cluster of 64 beads. Possible approaches to the experimental observation of the solid-state for an isolated chain are discussed. (C) 1997 American Institute of Physics. [S0021-9606(97)52547-5]
\end{abstract}

\section{INTRODUCTION}

Homopolymer transitions have been the subject of renewed theoretical ${ }^{1-21}$ and experimental ${ }^{22-29}$ interest in recent years. These studies ${ }^{1-20,22-27}$ have focused primarily on the second-order-like (except for stiff chains ${ }^{21,30-32}$ ) coil-toglobule collapse transition. ${ }^{30-34}$ In a previous paper, ${ }^{35}$ we reported that in addition to the collapse transition, liquidsolid and solid-solid polymorphic transitions are found for a freely-jointed off-lattice homopolymer chain consisting of 64 monomers $(64 \mathrm{mer})$ with square-well interactions. Surprisingly, the liquid-solid disorder-order transition was found to be first-order. Although such complex homopolymer phase behavior had been suggested by theories ${ }^{36,37}$ and experiments, ${ }^{25,26,28,29}$ it so far had been observed only in relatively-esoteric homonuclear lattice-chain models (the self-attracting self-avoiding trail on the Sierpinsku gasket, ${ }^{38}$ the two-dimensional self-attracting self-avoiding loop, ${ }^{39}$ and the three-dimensional lattice with attractive two-body interactions and repulsive three-body interactions ${ }^{17}$ ).

This paper provides the details of our previous brief publication ${ }^{35}$ describing the results of the study of an isolated $64 \mathrm{mer}$ modeled as a freely-jointed square-well (SW) chain with flexible bond length that was simulated using the discontinuous molecular dynamics (DMD) technique $e^{40-45}$ in the canonical ensemble. It also presents results for the effect of chain length, SW diameter, and bond constraints on the observed homopolymer transitions. In addition to the flexiblybonded model, ${ }^{35}$ we also investigate chain models with a fixed bond length via Monte Carlo (MC) simulation methods. Finally, we compare the thermodynamic results for isolated 64mers with those for a cluster containing the same number of SW atoms. This is expected to provide a deeper understanding of the role played by bead-connectivity in homopolymer transitions. Moreover, it is of interest because it permits a comparison with the multitude of results that exist for clusters of Lennard-Jones (LJ) spheres. ${ }^{46-49}$

The equilibrium mean-squared radius of gyration, internal energy and constant-volume heat capacity are obtained for square-well chains of length 3-8, 12, 16, 32, and 64 at a square-well diameter $\lambda \sigma=1.5 \sigma$ where $\sigma$ is the square-well hard-core diameter. Comparing MC results with DMD results indicates that the thermodynamics of the flexible-bondlength model and that of the fixed-bond-length model are quantitatively the same except at low temperature. The multiple equilibrium transitions reported earlier for a $64 \mathrm{mer}$ are also found for chains of length $N=6$ to $N=64$ (the longest chain considered) as measured by the appearance of plateaus or peaks in the heat-capacity versus temperature curve. For an isolated $64 \mathrm{mer}$, decreasing the square-well diameter from $\lambda=1.5$ to 1.3 weakens the liquid-solid transition; for $\lambda$ $=1.5$, it is strongly first-order-like with a clear bimodal distribution in the instantaneous potential energy at the transition temperature, while for $\lambda=1.3$, the bimodal distribution becomes a unimodal distribution. The transition behavior of an isolated $64 \mathrm{mer}$ is found to be similar to that of a cluster of $64 \mathrm{SW}$ atoms; both exhibit multiple transitions and both have a first-order-like liquid-solid transition. The thermodynamics 
of a SW cluster is shown to be similar to that for $\mathrm{LJ}$ clusters $^{46-49}$ and for lattice trees and lattice animals. ${ }^{50,51}$

The paper is organized as follows. The models and simulation methods are described in Secs. II and III, respectively. The results are presented in Sec. IV and discussed in Sec. V. In the Appendix, the specific form of the weighted histogram method $^{52}$ used for obtaining thermodynamic properties is described.

\section{MODELS}

\section{A. The chain model}

The homopolymer is modeled as a freely-jointed squarewell chain. The interaction between two nonbonded beads $i$ and $j$ is given by a square-well potential

$$
u_{i j}(r)=\left\{\begin{array}{l}
\infty, \quad r<\sigma, \\
-\epsilon, \quad \sigma<r<\lambda \sigma, \\
0, \quad r>\lambda \sigma
\end{array}\right.
$$

and the interaction between two bonded beads $i$ and $i+1$ is given by an infinitely-deep square-well potential,

$$
u_{i, i+1}^{\text {bond }}(r)= \begin{cases}\infty, & r<(1-\delta) \sigma, \\ 0, & (1-\delta) \sigma<r<(1+\delta) \sigma, \\ \infty, & r>(1+\delta) \sigma .\end{cases}
$$

Here, $\sigma$ is the bead hard-core diameter, $\lambda \sigma$ is the square-well diameter, $\epsilon$ is the square-well depth, and the bond length is allowed to vary freely from $(1-\delta) \sigma$ to $(1+\delta) \sigma$ where $\delta$ is the bond-length-flexibility parameter. This flexible squarewell bond, sometimes called a "Bellemans" bond, ${ }^{53}$ is introduced to decouple multibody collisions into binary collisions between monomer beads or between a monomer bead and a solvent particle in the DMD simulation. ${ }^{53-55}$ The flexible bond-length parameter is set to $\delta=0.1$ throughout this paper, except for the MC simulations which use a fixed bond-length model ( $\delta=0$ ) to save computing time. Thus, the comparison of the DMD results with the MC results for chains of the same length reveals how bond length flexibility affects the properties of the chain.

The simulations focus on the case in which the squarewell diameter $\lambda \sigma$ is equal to $1.5 \sigma$ for chains of various lengths except for 64mers. A 64 mer is investigated for $\lambda$ $=1.3$ and 1.5. The choice of the $\lambda$ values reflects the fact that the reduced square-well diameter $\lambda$ for real molecules is around 1.4 , based on fitting of experimental second-virial coefficient data. ${ }^{56}$

The simple square-well chain model is chosen in this study for the following reasons. First, the model is a reasonably realistic off-lattice model. It takes into account three important polymer features: bead connectivity, excluded volume, and bead-bead attraction. This chain model has been used successfully to reproduce thermodynamic properties of alkanes ranging from methane to eicosane. ${ }^{57}$ Second, the model is computationally much less demanding than a model with a continuous interaction potential such as the LJ potential. (For a system of 25 square-well chain butane molecules, the DMD algorithm is about 40 times faster than the SHAKE algorithm). ${ }^{43}$
TABLE I. Character of polymer chain models.

\begin{tabular}{lllll}
\hline \hline Chain model & Lattice & Sticky & Square-well & Realistic \\
\hline Bead positions & Discrete & Continuous & Continuous & Continuous \\
Interaction & Contact $^{\mathrm{a}}$ & Contact & Discontinuous & Continuous \\
\hline \hline
\end{tabular}

${ }^{a}$ Only nearest neighbor interactions are included in model studies.

A square-well chain can be viewed as a model that bridges the gap between a simple lattice chain model and a "realistic', chain model. Table I summarized the differences and similarities among four major types of polymer models: lattice chains, sticky chains, ${ }^{58,59}$ square-well chains, and "realistic" chains. The square-well chains are more realistic than the lattice chains not only in that the beads are allowed to be located anywhere in space but also in that the beadbead interactions exist over a finite range. A sticky chain ${ }^{58,59}$ (nonbonded beads interacting with a Baxter sticky potential_an infinitely narrow and deep square well potential $^{60}$ ) can be viewed as a model that differs from the lattice model only in the possible positions of the beads.

\section{B. The cluster model}

A cluster is not stable in the vapor phase. In a LJ-cluster simulation, the atoms are usually constrained to be inside an impenetrable sphere, the center of which is located at the coordinates of the center of mass (i.e., the sphere moves with the center of the cluster). ${ }^{61}$ A typical radius for the sphere is $5 \sigma_{\mathrm{LJ}}$ for clusters of up to 55 particles. ${ }^{48,49}$ In this paper, we use a constraint potential that is somewhat different but is more convenient for the DMD simulation. A SW cluster can be viewed as a SW chain with a bond length that can vary freely from $\sigma$ to $\infty$. Thus, instead of using the Bellemans bond potential for chains [Eq. (2)], we employ

$$
u_{i, i+1}^{\text {bond }}(r)= \begin{cases}\infty, & r<\sigma, \\ 0, & \sigma<r<\left(1+\delta_{c}\right) \sigma, \\ \infty, & r>\left(1+\delta_{c}\right) \sigma .\end{cases}
$$

In other words, the thermodynamics of an unconstrained SW cluster is obtained in the limit $\delta_{c} \rightarrow \infty$. Equation (3) was first used by Rapport as a bond potential for a chain (with a small value of $\left.\delta_{c}\right){ }^{54,55}$ As in LJ clusters, the constraint parameter $\delta_{c}$ has to be large enough to allow free movement of the beads but small enough to prevent beads from moving so far away from each other that they cannot recombine within the lifetime of a simulation. We use $\delta_{c}=4$ for a SW cluster of 64 atoms. This value allows free movement of beads if the cluster radius of gyration is less than $2.5 \sigma$, or $R_{g}^{2} / N \sigma^{2}$ $<0.1$, which is the case for a reduced temperature $T^{*}$ $=k_{B} T / \epsilon<0.8$. Although a larger $\delta_{c}$ is appropriate for higher temperatures, the overall transition pattern is expected to be similar to that obtained at $\delta_{c}=4$. 


\section{SIMULATION METHODS}

\section{A. Monte Carlo simulation}

Equilibrium MC simulations are performed in the canonical ensemble (constant temperature, constant volume, and constant number of particles). The MC procedure is as follows. First, an initial configuration is generated from a self-avoiding random walk. Then, chain monomers (beads) are moved via the "translate-jiggle" algorithm of Dickman and $\mathrm{Hall}^{62}$ in which the beads along the chain are chosen sequentially and subjected to a move if a random number is greater than a predetermined fraction (e.g. 50\%). When a bead is subjected to a move, it undergoes a small independent random displacement in which the bond vector joining the chosen bead and its neighbor is normalized so that the bond length is unchanged. After the move, the rest of the chain is translated so as to reconnect the chain. The Metropolis algorithm ${ }^{63}$ is used to determine whether or not to accept this move. The step size of a moving bead and the fraction of beads allowed to move are chosen to be between $0.3-0.5 \sigma$ and $10 \%-50 \%$, respectively, so that the acceptance rate of each move is around $1 \%-10 \%$.

During the simulation, the statistical averages of the mean-squared radius of gyration $R_{g}^{2}$, reduced internal energy $E^{*}$ and the reduced heat capacity $C_{v}^{*}$ are calculated from the following equations: ${ }^{64}$

$$
\begin{aligned}
& R_{g}^{2}=\left\langle\frac{1}{N} \sum_{i=1}^{N}\left[\left(x_{i}-x_{c}\right)^{2}+\left(y_{i}-y_{c}\right)^{2}+\left(z_{i}-z_{c}\right)^{2}\right]\right\rangle_{\mathrm{cf}}, \\
& E^{*}=\frac{\langle E\rangle_{\mathrm{cf}}}{\epsilon}, \\
& C_{v}^{*} \equiv C_{v} / k_{B}=\beta^{2}\left[\left\langle E^{2}\right\rangle_{\mathrm{cf}}-\left(\langle E\rangle_{\mathrm{cf}}\right)^{2}\right],
\end{aligned}
$$

where \langle\rangle$_{\text {cf }}$ denotes the configurational average, $N$ is the chain length, $x_{i}, y_{i}$, and $z_{i}$ are the coordinates for bead $i$ and $x_{c}$, $y_{c}$, and $z_{c}$ are the center of mass coordinates for the chain, $E$ denotes the internal energy, $\beta=1 / k_{B} T, k_{B}$ is the Boltzmann constant, and $T$ is the temperature. Five independent runs of lengths ranging from $10^{6}$ to $10^{11}$ Monte Carlo steps are conducted to obtain a $95 \%$ confidence limit for averages. The simulation code has been tested against Taylor's exact results ${ }^{15}$ for the internal energy, heat capacity, and radius of gyration of an isolated square-well tetramer.

\section{B. Discontinuous molecular dynamics}

Molecular dynamics simulation algorithms for chains interacting with discontinuous potentials such as hard-sphere and square-well potentials are different from those for chains interacting with soft potentials such as LJ interactions. Unlike soft potentials, discontinuous potentials only exert forces when particles collide. The binary collision dynamics for discontinuous potentials can be solved exactly. Thus, the DMD algorithm ${ }^{40-43,45}$ involves searching for the next collision time and collision pair, moving all beads for the duration of the collision time, and then calculating the velocity changes of the colliding pair.
Molecular dynamics simulations are often performed in a microcanonical ensemble, that is, an ensemble containing a constant number of particles, constant total energy, and constant volume. ${ }^{65}$ The details of the use of the DMD algorithm for the case of a square-well chain in a microcanonical ensemble can be found in earlier work. ${ }^{40-43,45}$ In a constant energy ensemble, however, a short isolated chain is not an ergodic system at low energies because the chain can be trapped permanently in a low-energy configuration if its kinetic energy is not high enough to overcome the energy barrier. This problem may be remedied by placing the isolated chain in a constant-temperature bath. The collision between the bath particles and the chain can help the chain to get out of low energy traps. For this reason, we simulate the isolated square-well chain in a "canonical" ensemble, that is, an ensemble with constant number of particles, $N$, and constant temperature, $T$; for an isolated homopolymer, there is no volume constraint.

Current constant-temperature MD techniques include velocity-scaling ${ }^{66}$ stochastic collision methods,${ }^{67}$ constraint methods ${ }^{68}$ extended system methods ${ }^{69}$ and various hybrid MC methods. ${ }^{70-73}$ The Andersen stochastic collision method $^{67}$ is best suited for the present case. In the Andersen method, an isolated chain is immersed in a constant temperature bath of imaginary (ghost) particles, a system that can be easily handled by DMD techniques. To our knowledge, this is the first application of the Andersen method to DMD simulations.

The basic idea of the Andersen method is that particles experience random collisions with imaginary heat-bath particles (ghost particles) which do not appear explicitly in simulations. The Andersen method can be incorporated into DMD techniques by introducing a new type of collision-the "bead-ghost" collision-in addition to core, bond, and square-well collisions. The "collision free" time for a particle satisfies an exponential distribution. ${ }^{74}$ Thus, the time at which a "bead-ghost" collision occurs is calculated from an exponential-distribution-random-number generator ${ }^{65,67}$

$$
P(t)=\nu e^{-\nu t},
$$

where $t$ is the time since the previous ghost or real collision and $\nu$ is the mean bead-ghost collision rate. Most bead-ghost collisions occur at $t<1 / \nu$.

Assuming that the ghost heat-bath particles are hardspheres which have the same size and mass as the polymer bead, we can calculate the mean collision rate $\nu$ with a given bead from the kinetic theory of gases ${ }^{74}$

$$
\nu=n_{g} \sigma^{2} \sqrt{16 \pi k_{B} T / m},
$$

where $n_{g}$ is the number density of ghost particles and $m$ is the mass of ghost particles or polymer beads. Equation (8) is based on the assumption that each polymer bead is connected to its own heat bath. For a single chain in a real solvent, the bead-solvent particle collision rate $\nu$ is different for each bead. For example, the bead-solvent collision rate for the middle bead of a trimer is expected to be smaller than that for the two end beads because the end beads shield the middle bead. However, assigning different collision rates to 
each bead does not alter the equilibrium results though it is expected to change the kinetics. Thus, we use the same beadghost particle collision rate $\nu$ for all beads in this paper. The value of the bead-ghost particle collision rate $\nu$ also does not affect the equilibrium results. ${ }^{67}$ It only changes the rate at which the polymer beads explore configurational space and reach equilibrium. For dynamic properties, if a collision rate is too high, the simulation generates exponentially decaying single-particle time correlation functions ${ }^{75}$ but otherwise ghost collisions have only a small effect on time correlations. ${ }^{76}$ In general, we find that reduced ghost densities, $n_{g}^{*}=n_{g} \sigma^{3}$, ranging from 0.05 to 0.5 yield a number of ghost collisions that is $1 \%-10 \%$ of the total number of collisions; this is adequate to generate a highly accurate constant-temperature trajectory. The relative differences between the average temperatures among independent runs are less than $1 \%$. The fluctuation of temperatures (kinetic energy, KE) also accurately reproduces the kinetic contribution to the heat-capacity (ideal heat capacity), i.e., $C_{v}^{i d}$ $=\partial\langle\mathrm{KE}\rangle / \partial T=\left(\left\langle\mathrm{KE}^{2}\right\rangle-\langle\mathrm{KE}\rangle^{2}\right) / k_{B} T^{2}=3 N k_{B} / 2$, where $\langle\mathrm{KE}\rangle=3 N k_{B} T / 2$. [It should be noted that the heat-capacity $C_{v}$ reported in this paper is the excess heat-capacity over the ideal term $C_{v}^{i d}$; that is, $C_{v}=C_{v}^{\text {tot }}-3 N k_{B} / 2$, where $C_{v}$ $=\partial\langle E\rangle / \partial T$ and $C_{v}^{\text {tot }}=\partial\left\langle E_{T}\right\rangle / \partial T$ with $\langle E\rangle$, the average internal (potential) energy and $\left\langle E_{T}\right\rangle$, the average total energy.]

In a constant-temperature DMD simulation, the ghost particles mimic real solvent particles. The randomlygenerated bead-ghost collision time for each bead based on Eq. (7) is recalculated only if the velocity of that bead is changed due to collision (core, bond, well or ghost collision). If a bead-ghost collision occurs, the velocity of the bead that collides with a ghost particle is chosen afresh from the Maxwell-Boltzmann distribution at the simulated temperature. Thus, the constant-temperature DMD algorithm differs from the constant-energy DMD algorithm ${ }^{40-43,45}$ only in the addition of a new collision type: the bead-ghost particle collision.

Various time-saving techniques that are used in the constant-energy DMD algorithm ${ }^{45}$ can also be applied to the constant-temperature algorithm. These include the singleevent time list, ${ }^{65}$ the neighbor list, ${ }^{77}$ the link list, ${ }^{78}$ the binary tree minimum-time search, ${ }^{42}$ and the delayed position update. ${ }^{79}$ The single-event time list is found to be very useful for an isolated short chain $(N<100)$. In the single-event time list ${ }^{65}$ the times of the soonest-to-occur collision for each particle are stored. As a result, only the collision times of beads involving the last colliding pair needs be updated. We find that for an isolated square-well 64mer the computing time is reduced by a factor of 10 by the use of the singleevent time list. The other techniques are not used in this work because they are suitable only for systems of 300 or more particles. ${ }^{43}$

Representative thermodynamic quantities for an isolated chain are the average internal energy and heat capacity. In the DMD simulation, the reduced internal energy $E^{*}$ and the average squared internal energy $\left\langle E^{2}\right\rangle_{t}$ can be obtained by taking time averages over the equilibrium trajectory ${ }^{65}$

$$
\begin{aligned}
& E^{*}=\langle E\rangle_{t} / \epsilon=\frac{1}{t_{\mathrm{eq}} \epsilon} \int_{0}^{t_{\mathrm{eq}}} E(t) d t=\frac{\sum_{i} E_{i} t_{i}}{\epsilon \sum_{i} t_{i}}, \\
& \left\langle E^{2}\right\rangle_{t}=\frac{1}{t_{\mathrm{eq}}} \int_{0}^{t_{\mathrm{eq}}} E^{2}(t) d t=\frac{\sum_{i} E_{i}^{2} t_{i}}{\sum_{i} t_{i}},
\end{aligned}
$$

where $t_{\text {eq }}$ is the equilibrium simulation time $\left(=\Sigma_{i} t_{i}\right)$, the summation is over all collisions after equilibration, $E_{i}$ and $t_{i}$ are the internal energy of the system and the time between the $(i-1)$ th collision and the $i$ th collision, respectively. The replacement of the integrals in Eqs. (9) and (10) by summations is exact because the internal energy of an isolated chain does not change between collisions. In a constanttemperature simulation, the constant-volume heat capacity can be obtained either from the temperature derivative of the energy or from the fluctuation in the internal energy, ${ }^{64}$ i.e.,

$$
C_{v}^{*}=\frac{1}{k_{B}} \frac{\partial\langle E\rangle_{t}}{\partial T}=\frac{\left(\left\langle E^{2}\right\rangle_{t}-\langle E\rangle_{t}^{2}\right)}{k_{B}^{2} T^{2}} .
$$

Unlike $C_{v}$ and $E$, the squared radius of gyration varies between collisions. To save computing time, the squared radius of gyration is calculated via the configurational average [Eq. (4)] rather than the time average; a configuration from the DMD simulation is sampled every 100 or 1000 collisions.

The partition function $Z$ can also be obtained via DMD simulations. Since square-well chain models have discrete energy levels, the partition function $Z$ can be expressed as sum over all energy levels as follows ${ }^{64}$

$$
Z=\sum_{k} g_{k} e^{-\beta E_{k}},
$$

where $E_{k}$ and $g_{k}$ are the energy and degeneracy factor for the energy level $k$, respectively. The degeneracy factor $g_{k}$ is the sum of contributions from the various configurations that have the same energy. The contribution of each configuration depends on the volume of configurational space in which the chain is free to move without changing its energy. The partition function $Z$ can be calculated for any temperature if the temperature-independent degeneracy factors $g_{k}$ are known. If all energy levels have statistically significant populations at one temperature, it is possible to extract $g_{k}$ from the probability distribution in simulation runs at that temperature. ${ }^{80}$ For chains with more than 5 beads, simulations at one temperature do not adequately sample all states. Thus, the weighted histogram method ${ }^{52}$ was used to extract $g_{i}$ from simulations at several temperatures. In this work, the Ferrenberg-Swendsen equation for the weighted histogram method is modified slightly in that the equation is based on a normalized population rather than on an unormalized one. The details can be found in the Appendix.

Once the partition function is known, thermodynamic quantities can be calculated from the relations ${ }^{64}$

$$
\begin{aligned}
& A^{*}=-T^{*} \ln Z, \\
& E^{*}=\left(T^{*}\right)^{2} \frac{\partial \ln Z}{\partial T^{*}},
\end{aligned}
$$




$$
C_{v}^{*}=\frac{\partial E^{*}}{\partial T^{*}}
$$

where $T^{*}\left(=k_{B} T / \epsilon\right)$ is the reduced temperature and $A^{*}$ ( $=A / \epsilon$ ) is the reduced Helmholtz free energy.

Another important quantity is the bead-bead rms relative distance fluctuation, given by

$$
\Delta_{B}=\frac{2}{N(N-1)} \sum_{i<j} \frac{\sqrt{\left(\left\langle r_{i j}^{2}\right\rangle_{\mathrm{cf}}-\left\langle r_{i j}\right\rangle_{\mathrm{cf}}^{2}\right)}}{\left\langle r_{i j}\right\rangle_{\mathrm{cf}}}
$$

where $r_{i j}$ is the distance between beads $i$ and $j$. For a homopolymer, $\Delta_{B}$ as defined above contains a negligible contribution from bonded beads $(\sim 2 \delta / N=0.003)$. The rms bead-bead distance fluctuation $\Delta_{B}$ is used to characterize the liquid-solid-like transition in clusters or other systems without crystalline order. ${ }^{46}$ It is related to a disorder parameter $\Delta_{L}$ which compares the atomic vibrational amplitude $\left\langle\Delta r^{2}\right\rangle^{1 / 2}$ with the lattice constant $a$ of a crystal. According to Lindemann's rule, ${ }^{81,82}$ there is a critical value of $\Delta_{L}$ above which a solid melts into a liquid since the vibrational amplitude can no longer increase without damaging the solid lattice. This critical value of $\Delta_{L}$ for simple infinite solids and finite clusters is found to be $0.1-0.15$ for a wide range of substances with various interaction potentials and crystal structures. ${ }^{83-86}$ It has been shown that the critical ratio for the distance fluctuation $\Delta_{B}$ is also around 0.1 for $\mathrm{LJ}$ clusters. ${ }^{46,48}$

Application of Lindemann's rule to a finite irregular system requires evaluation of ${ }^{86}$

$$
\Delta_{L_{i}}=\frac{\left\langle\Delta r_{i}^{2}\right\rangle_{\mathrm{cf}}^{1 / 2}}{a}, \Delta_{L}=\left(\sum_{i} \Delta_{L_{i}}^{2} / M\right)^{1 / 2},
$$

where $a=\sigma$, the near neighbor distance, $\mathbf{r}_{i}$ is the position of bead $i,\left\langle\Delta r_{i}^{2}\right\rangle_{\mathrm{cf}}\left[=\left\langle\left(r_{i}-\left\langle r_{i}\right\rangle_{\mathrm{cf}}\right)^{2}\right\rangle_{\mathrm{cf}}\right]$ is the mean-squared fluctuation, and $\Delta_{L_{i}}$ is the Lindemann disorder parameter for an individual bead. In the calculation of $\left\langle\Delta r_{i}^{2}\right\rangle_{\mathrm{cf}}$, the translations and rotations of all configurations respect to the first configuration are removed by shifting the center of mass coordinates to the origin and minimizing the root mean-squared deviation through finite rotations. ${ }^{87}$ The disorder parameter $\Delta_{L}$ for the whole chain or a part of chain is obtained from the root-mean-squared average of $\Delta_{L_{i}}$ over the chosen $M$ beads.

The radial and bond-angle distribution functions are important indicators of the intramolecular structure of a chain. The radial and bond-angle distribution functions are calculated via a simple histogram method. ${ }^{65}$

The initial configurations of the DMD simulations are obtained from self-avoiding random walks and the initial velocities are obtained from random numbers generated from the Maxwell-Boltzmann distribution at the simulation temperature. The equilibrium results are obtained from five independent runs (different initial configurations and velocities) which last from 6 million to 1 billion collisions depending on the temperature and chain length. The first half of the collisions (equilibration) are discarded. We increase the total number of collisions until the range of the $95 \%$ confidence limit on the heat capacities based on five inde-

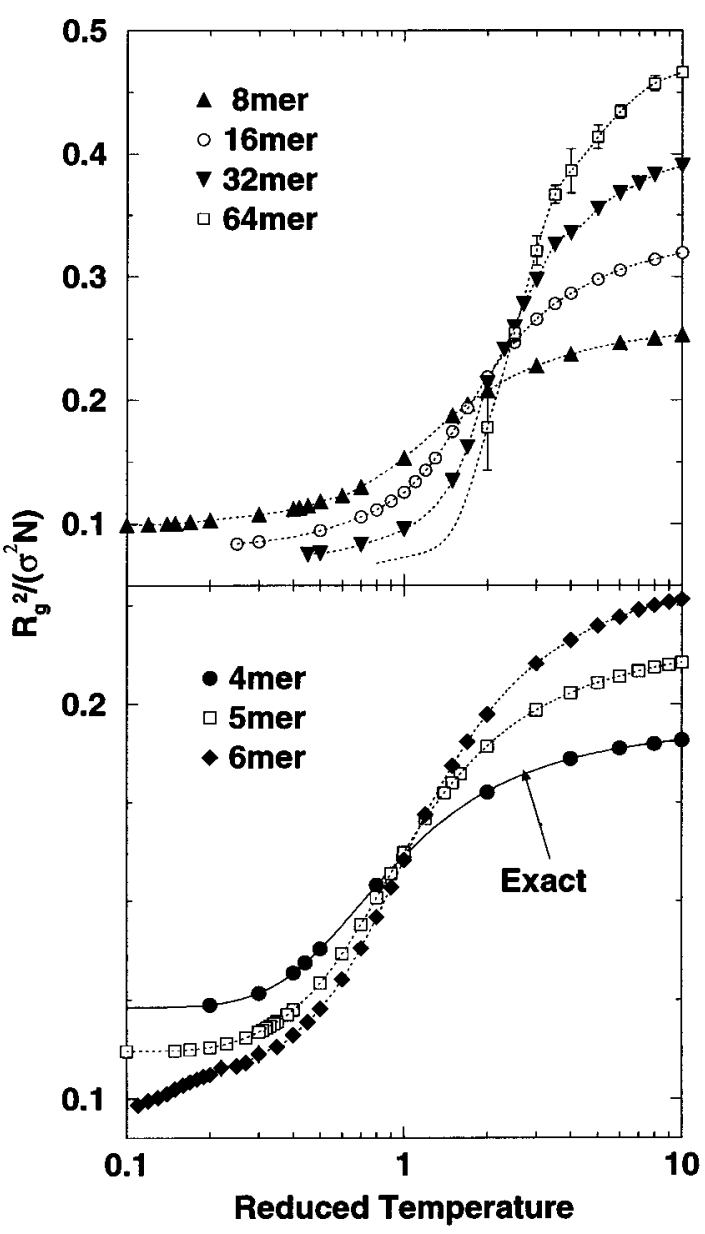

FIG. 1. Monte Carlo simulation results for the normalized squared radius of gyration $R_{g}^{2} /\left(\sigma^{2} N\right)$ as a function of reduced temperature $T^{*}\left(=k_{B} T / \epsilon\right)$ for isolated square-well chains of different lengths. The exact result for an isolated 4mer (Ref. 15) is shown as a solid line. Error bars are less than the size of the points except as shown; dotted lines are results from spline fit.

pendent runs (student-T test) is reasonably small or the total number of collisions reaches 1 billion. For low temperature simulations, it is found necessary to anneal the random initial configuration from a high temperature (for example, $T^{*}=1$ ) before performing an equilibrium simulation to avoid the system being trapped in local minima.

\section{RESULTS}

\section{A. MC results for chains of fixed-bond lengths}

Figure 1 plots the Monte Carlo results for the normalized squared radius of gyration $R_{g}^{2} /\left(\sigma^{2} N\right)$ as a function of reduced temperature $T^{*}$ for chains of length $N=4,5,6,8,16$, 32 and 64 and reduced square-well diameter $\lambda=1.5$. The curves are sigmoidal in shape which indicates the presence of a coil-globule or collapse transition. The MC simulation results for an isolated $4 \mathrm{mer}$ are in excellent agreement with the exact results obtained by Taylor. $^{15}$ The Flory $\Theta$ temperature for the infinitely-long square-well chain can be estimated from the position of the intersection of the $R_{g}$ curves of the three longest chains (16mers, 32mers, and 64 mers) that are simulated. ${ }^{5}$ Figure 1 shows $T_{\Theta}^{*} \approx 2.5$. An- 


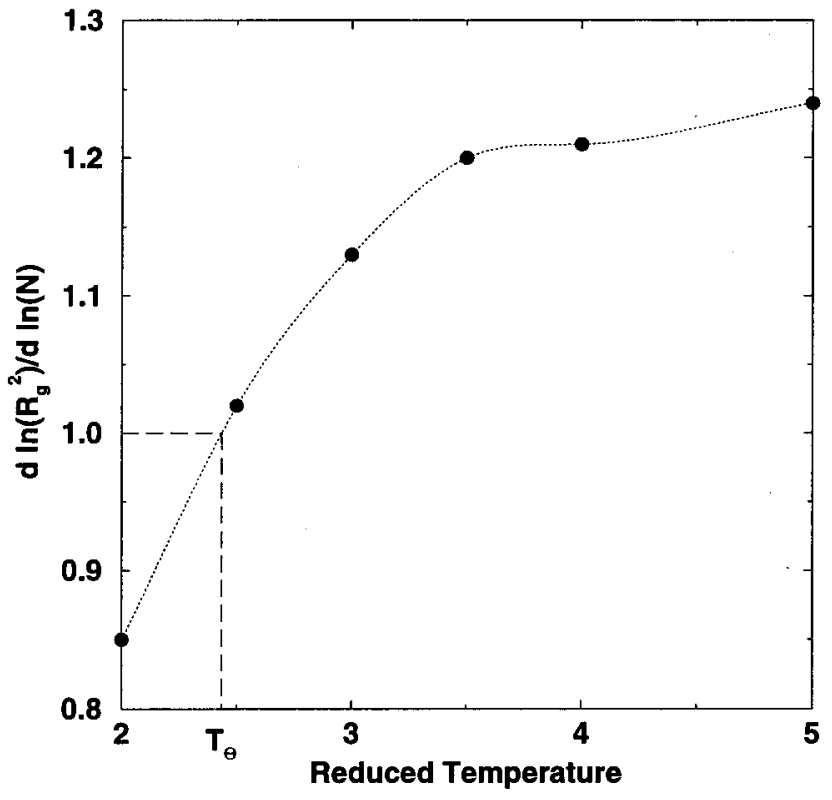

FIG. 2. Average value of slope of $\ln \left(R_{g}^{2}\right)$ vs $\ln (N)$ over the chain lengths 16 , 32 , and 64 at various reduced temperature $T^{*}$; the dotted line results from a spline fit and the dashed line indicates the approximate location of $T_{\Theta}^{*}$.

other method for estimating the $\Theta$-temperature is to calculate the temperature at which $R_{g}^{2} \propto N$ as $N \rightarrow \infty ; 88$ i.e., at the $\Theta$ temperature, the slope of $\ln \left(R_{g}^{2}\right)$ versus $\ln (N)$ should approach 1 for long chains. Figure 2 shows the average slopes of $\ln \left(R_{g}^{2}\right)$ versus $\ln (N)$ over chain lengths ranging from $N=16$ to $N=64$ at different temperatures. According to this criterion, the $\Theta$-temperature is again around 2.5. More accurate assessment of the $\Theta$-temperature requires data on the radius of gyration for longer chains. The $\Theta$-temperature estimated here is in close agreement with the value of $T_{\Theta}^{*} \approx 3$ determined from the temperature at which the second virial coefficient of square-well chains is zero. ${ }^{89}$ Based on the data for the radius of gyration alone, it appears that the homopolymer transition at $T_{\Theta}^{*} \sim 2.5$ is a well-defined single-stage collapse process and that there are no transitions below that temperature.

The heat capacity results for the isolated square-well chains show a much richer and more complex transition pattern than the radius of gyration curves. Figure 3 plots the reduced heat capacity per bead, $C_{v}^{*} / N$, as a function of the reduced temperature $T^{*} . C_{v}^{*}$ is evaluated using the fluctuation equation here [Eq. (6)]; $C_{v}^{*}$ determined from $\partial E^{*} / \partial T^{*}$ is essentially the same. Again, the MC simulation results for the heat capacity of an isolated 4mer agree with Taylor's exact results, ${ }^{15}$ exhibiting a well-defined single peak. However, as the chain length increases, this peak flattens out for 5 mers and then splits into two peaks for 6mers. The two peaks shown in the $C_{v}$ versus $T^{*}$ curve for 6mers clearly signal the existence of two equilibrium transitions. As the chain length increases further, the high-temperature peak flattens out into a plateau at $C_{v}^{*} / N \sim 1.25$ for chain lengths of $7,8,12,16,32$; the position of the plateau is at a higher temperature for longer chains. Similar chain-length depen-

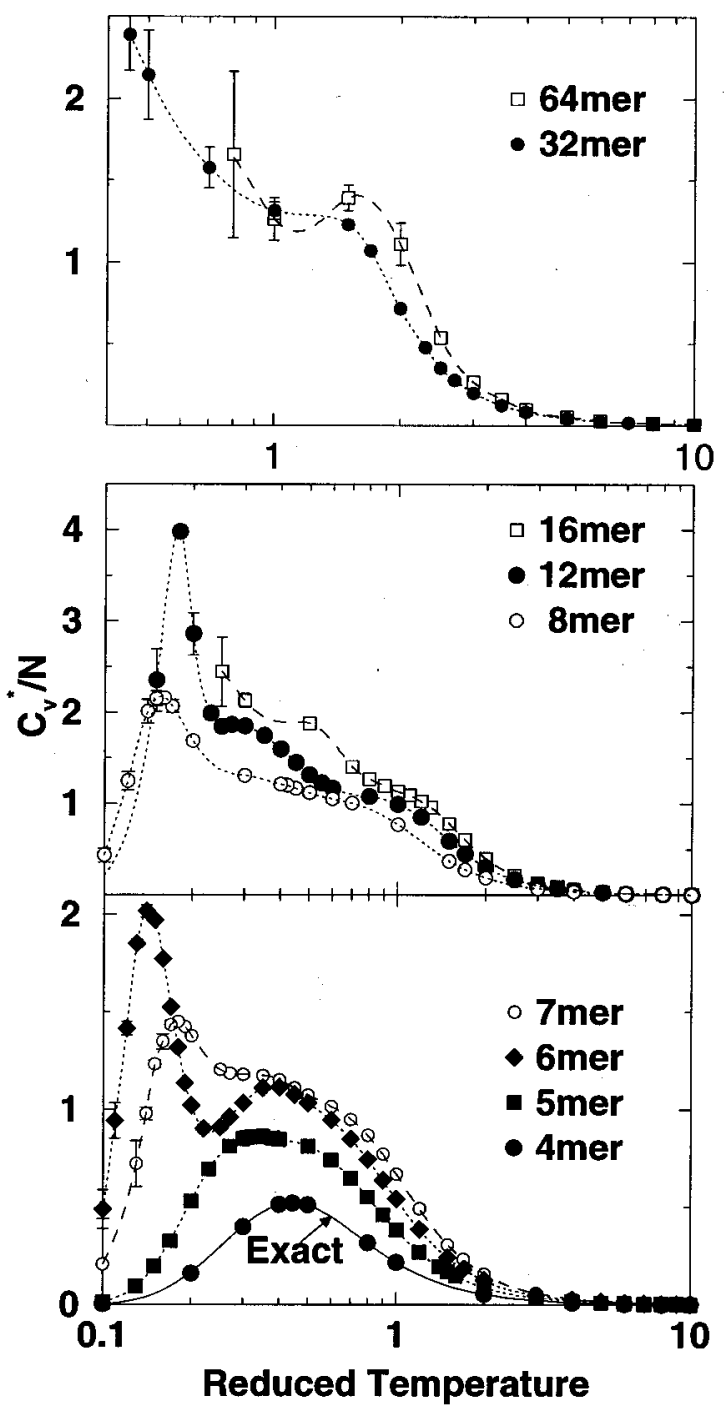

FIG. 3. MC results for the reduced heat capacity per segment $C_{v}^{*} / N$ $\left[=C_{v} /\left(k_{B} N\right)\right]$ as a function of reduced temperature for isolated square-well chains of different lengths. The exact result for an isolated 4mer (Ref. 16) is shown as a solid line. Error bars are less than the size of points except as shown; dotted and dashed lines are results from spline fit.

dence is also observed for the temperature location of collapse transition as indicated by the $R_{g}^{2}$ curves. As the chain length increases to $N=64$, the plateau again becomes a peak centered at $T^{*}=1.5$. The low-temperature peak, on the other hand, persists as chain length increases. Since the low temperature peak is the heat capacity maximum, comparison of Figs. 3 and 1 indicates that at least for $6 \mathrm{mer}, 7 \mathrm{mer}, 8 \mathrm{mer}$ and $12 \mathrm{mer}$, the heat capacity maximum does not signal polymer collapse since the radii of gyration do not vary significantly with $T^{*}$ around the transition temperature. At the chain length of 12 , there is an additional peak centered at $T^{*}$ $=0.27$, suggesting that an isolated $12 \mathrm{mer}$ experiences three transitions. Similar behavior is also found for the $16 \mathrm{mer}$; its heat capacity has two plateau values at temperatures higher than the heat-capacity-maximum temperature.

To take a closer look at the transition behavior for short chains, we examine the transition of an isolated 6mer in de- 


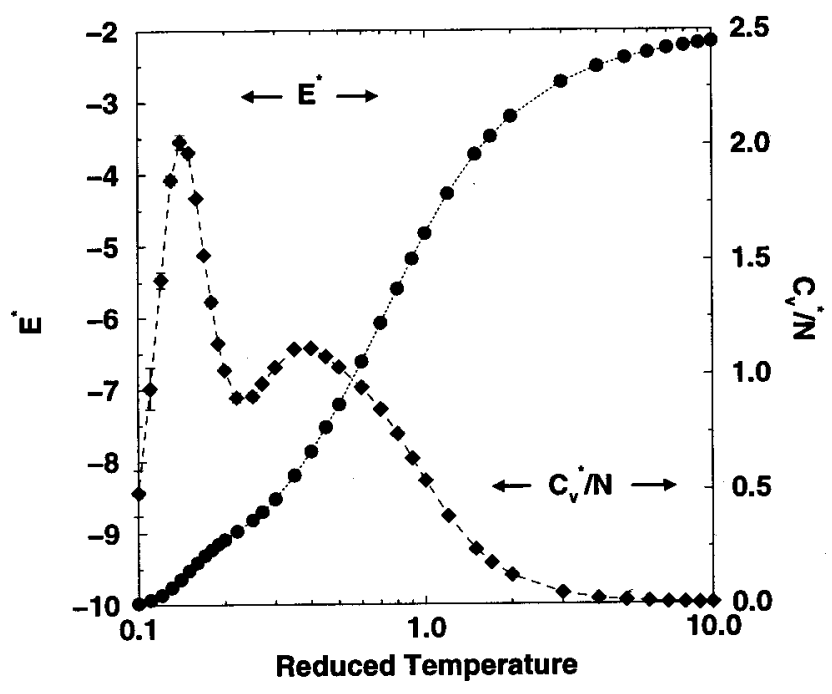

FIG. 4. MC results for reduced internal energy $\left(E^{*}=\langle E\rangle / \epsilon\right)$ and reduced heat capacity per segment as a function of reduced temperature for an isolated square-well 6mer; dotted and dashed lines are results from spline fit.

tail in Fig. 4, which plots the energy and heat capacity as a function of reduced temperature. At low temperatures, the simulation results for the internal energy approach -10 , which corresponds to the maximum number of square-well contacts possible for an isolated $6 \mathrm{mer} .{ }^{15}$ As the figure shows, the low-temperature peak in the heat capacity is located between the average energy values of -9 and -10 . Thus, the low-temperature peak $\left(C_{v}\right.$ maximum $)$ signals the transition from the energy level of -9 to the energy level of -10 , while the high temperature peak signals the continuous collapse transition from high energy states to the energy level of -9. It appears that as the temperature decreases, an isolated 6 mer first collapses to the energy level of -9 and then, as the temperature is further reduced, the chain reorganizes itself into the lowest energy level of -10 . We use the word "reorganize" for the low-temperature transition since this transition occurs within a collapsed structure and involves a much smaller change in radius of gyration than the high temperature transition (see Fig. 1).

For chains with lengths of 16, 32 and 64, the MC simulations at low temperatures do not converge sufficiently within a reasonable computational time. This is because the "translate-jiggle" moving algorithm, ${ }^{62}$ like many others,${ }^{90}$ is unable to handle compact high-density chain configurations. Although other MC moving algorithms such as the reptation method, ${ }^{91,92}$ the continuum-configurational-bias method ${ }^{93-95}$ and the concerted-rotation method ${ }^{96,97}$ might improve the sampling efficiency for low-temperature compact configurations, we use the DMD technique to obtain converged results in this work.

\section{B. DMD Results for chains with flexible bond lengths $(\lambda=1.5)$}

\section{Trimer}

The DMD code can be tested against the exact results for an isolated flexibly-bonded square-well trimer. ${ }^{98}$ In Fig. 5,

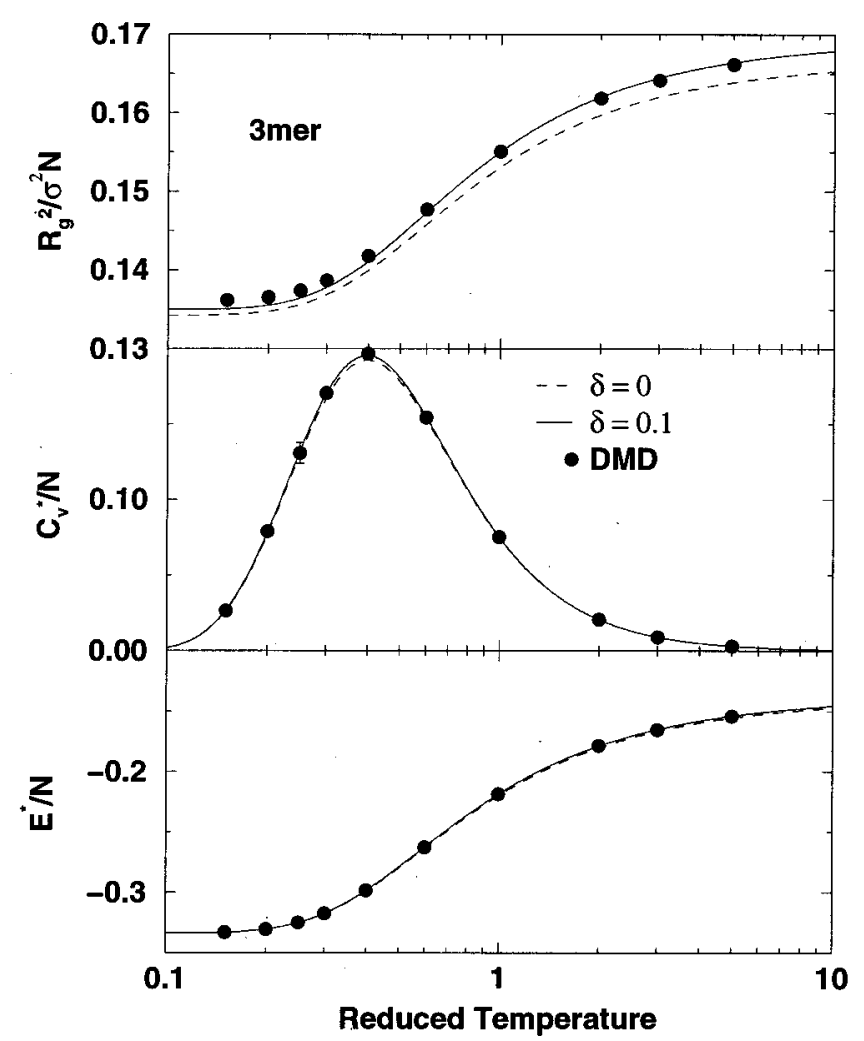

FIG. 5. The reduced time-averaged internal energy $E^{*}=(\langle E\rangle / \epsilon)$ per bead, the reduced heat capacity $C_{v}^{*}\left(=C_{v} / k_{B}\right)$ per bead, and the reduced configurationally-averaged squared radius of gyration $R_{g}^{2} / \sigma^{2}$ per bead for an isolated freely-jointed square-well trimer are plotted as a function of reduced temperature $T^{*}=\left(k_{B} T / \epsilon\right)$. Exact results for the flexibility parameter $\delta=0.1$ (Ref. 98) and $\delta=0$ (Ref. 15) (the rigid-bond-length chain limit) are shown as solid line and dashed line, respectively. The constant-temperature DMD simulation is conducted at $\delta=0.1$ (filled circles). The square-well diameter $\lambda \sigma=1.5 \sigma$. Error bars are smaller than the size of points except as shown.

the reduced time-averaged internal energy $E^{*}$ per bead, the reduced heat capacity $C_{v}^{*}$ per bead, and the reduced configurationally-averaged squared radius gyration $R_{g}^{2} / \sigma^{2}$ per bead for an isolated trimer with bond-length flexibility parameter $\delta=0.1$ and square-well diameter $\lambda=1.5$ are plotted as a function of reduced temperature $T^{*}$. The constanttemperature DMD simulation results (denoted by filled circles) are in perfect agreement with the exact results ${ }^{98}$ for the thermodynamic properties $E^{*}$ and $C_{v}^{*}$ (denoted by solid lines).

There is slight difference, however, between the simulation results and the exact results for the radius of gyration (Fig. 5). The simulation results for the radius of gyration are slightly larger than the exact results at low temperature and are very slightly smaller than the exact results at high temperature. We attribute this small discrepancy to biased configuration sampling. The configurations, which were sampled from simulations every 100 or 1000 collisions (see Sec. III B), are at a position where a collision has occurred because that determines the steps of a DMD simulation. This configurational bias will lead to an overestimation of $R_{g}$ at low temperatures and an underestimation at high tempera- 
tures because of inclusions of too many configurations of beads at the boundary of the square-well interaction. Such bias is most severe for a trimer because one or two out of the three beads have collided. To verify this bias, we modified the program in that polymer beads are allowed to move over a random time interval that is less than the next collision time before the configuration is selected for the average. With this modification, we find that the agreement for the radius of gyration between the simulation results and the exact results improves. However, we do not employ this modification to save computing time. The bias introduced is very small for the trimers and is expected to be even smaller for longer chains.

To illustrate the difference between the trimer with fixed bond lengths and the trimer with flexible bond lengths, we also plot the exact result ${ }^{15}$ for a chain with a fixed bond length (denoted as dashed line) in Fig. 5. It shows that the difference of the $R_{g}^{2}$ and thermodynamic results between the two chain models is small but detectable in an isolated trimer. $^{98}$

\section{Octamer (8mer)}

The thermodynamic properties and radius of gyration for an isolated square-well 8mer are shown as a function of reduced temperature in Fig. 6. The MC simulation results ( $\delta=0$, denoted as open circles) are compared with the constant-temperature DMD results $(\delta=0.1$, denoted as filled circles). The MC and MD results have the same qualitative features for all quantities investigated although there are quantitative differences at low temperature. The internal energy and radius of gyration for the flexibly-bonded chain (DMD results) are slightly smaller than the results for the rigidly-bonded chain (MC results) at low temperature. These energy and size reductions are caused by the ability of a flexibly-bonded chain to reach a lower energy level than the rigidly-bonded model since neighboring beads in a flexiblybonded chain experience smaller hard cores than those in a rigidly-bonded chain. As temperature decreases, the reduced internal energy (or the negative of the average number of square-well overlaps) approaches -17 for the rigidlybonded chain but approaches -18 for the flexibly-bonded chain with $\delta=0.1$. (For an even larger $\delta$, the absolute value of the internal energy of a flexibly-bonded chain could reach 21 , the maximum number of allowable square-well overlaps for an 8mer.) At high temperature, the internal energy and radius of gyration seem to be independent of bond flexibility. Evidently, the tendency of flexibly-bonded beads to be further apart at high temperature ${ }^{98}$ is essentially cancelled by the ability of the flexibly-bonded chain to reach a lower energy level than can the rigidly-bonded chain.

The heat-capacities for the flexibly-bonded and rigidly bonded 8mers are similar at high temperatures but the flexibly-bonded chain has a stronger low-temperature peak than the rigidly-bonded chain. The stronger peak is caused by the ability of the flexibly-bonded chain to reach a lower energy than the rigidly-bonded chain. The heat-capacity of the isolated flexibly-bonded 8 mer has a peak at $T^{*} \approx 0.22$

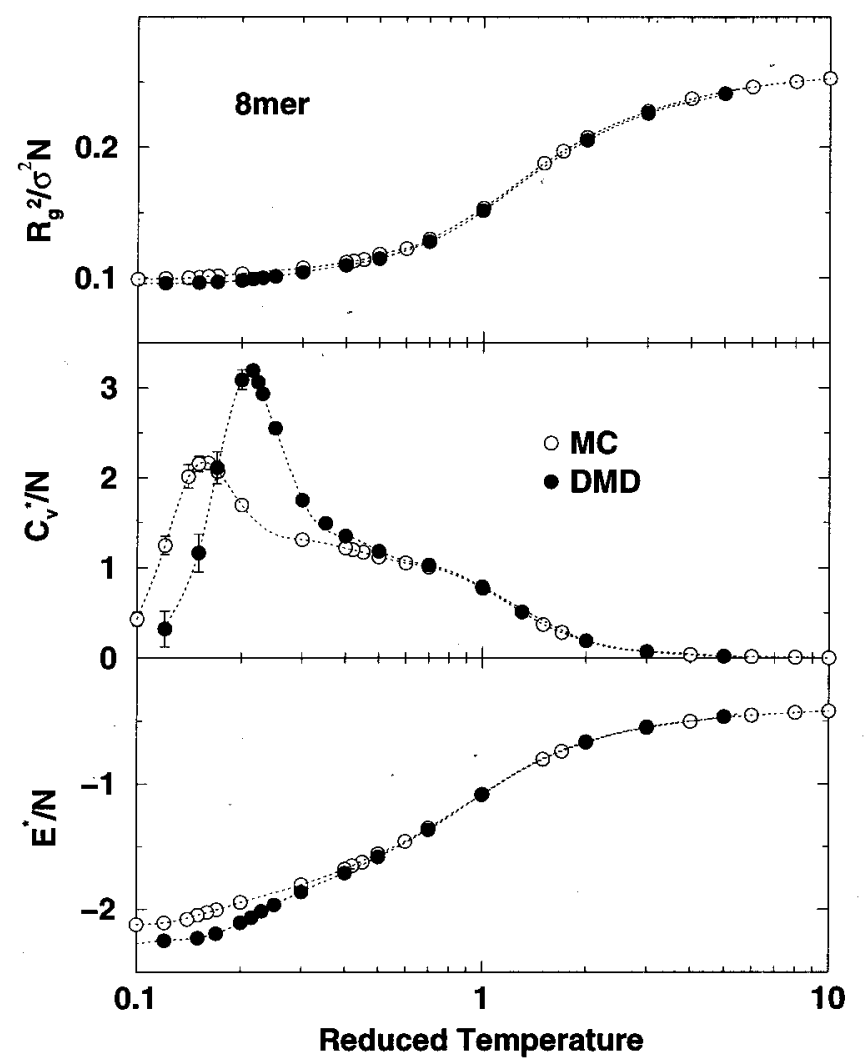

FIG. 6. The reduced time-averaged internal energy $E^{*}=(\langle E\rangle / \epsilon)$ per bead, the reduced heat capacity $C_{v}^{*}\left(=C_{v} / k_{B}\right)$ per bead, and the reduced configurationally-averaged squared radius of gyration $R_{g}^{2} / \sigma^{2}$ per bead for an isolated freely-jointed square-well $8 \mathrm{mer}$ are plotted as a function of reduced temperature $T^{*}=\left(k_{B} T / \epsilon\right.$ ). The $\mathrm{MC}$ simulation result for $\delta=0$ (open circles) is compared with the constant-temperature DMD simulation results for $\delta=0.1$ (filled circles). The square-well diameter $\lambda \sigma=1.5 \sigma$. Error bars are smaller than the size of points except as shown; dotted lines are results from spline fit.

and a plateau at $T^{*} \approx 0.6$. The plateau temperatures for the flexibly-bonded and rigidly-bonded chains are the same but the heat-capacity-vs-temperature peak for the flexiblybonded model is at a higher temperature than the peak for the rigidly-bonded model. In both the MC and DMD results, the heat-capacity peak corresponds to a temperature range in which the radius of gyration is nearly constant. This suggests that the heat-capacity peak does not signal a polymer collapse transition..$^{99,100}$ The plateau is the more likely candidate for the collapse transition which is characterized by a sudden reduction of the chain size. Figure 6 also suggests that the two transitions (represented by the plateau and the peak) for a square-well $8 \mathrm{mer}$ get closer together as the bond flexibility parameter $\delta$ changes from 0 to 0.1 .

\section{16mer}

The equilibrium results for an isolated $16 \mathrm{mer}$ are shown in Fig. 7. Unlike the isolated 8mer, MC simulation is unable to provide accurate low-temperature data within a reasonable computing time. As was the case for 8 mers, bond-length flexibility has little or no effect on thermodynamic properties at high temperature. At low temperature, the internal energy 


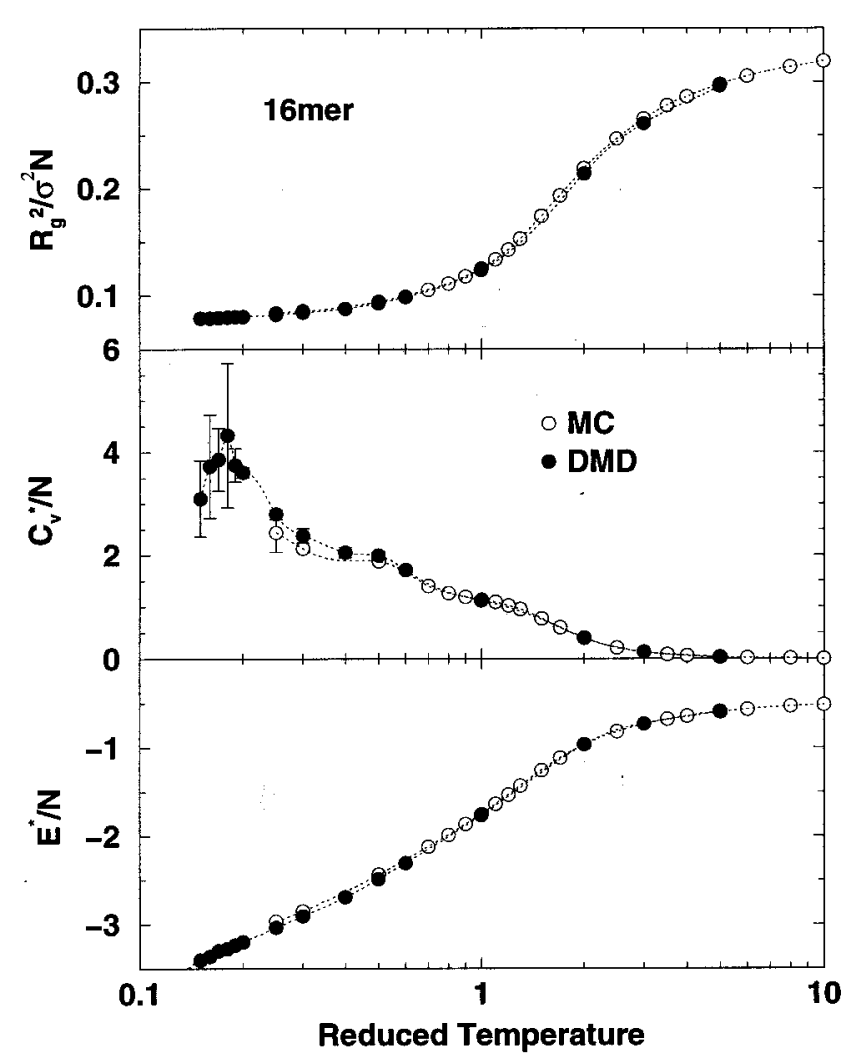

FIG. 7. As in Fig. 6 except for an isolated 16mer. For the radius of gyration, two MC results at $T^{*}=0.25$ and 0.3 are nearly hidden due to overlap with the DMD results.

and radius of gyration for a flexibly-bonded chain are slightly lower than those for a rigidly-bonded chain based on the available data. For an isolated square-well 16mer, two plateaus appear on both the MC and DMD heat-capacity-vstemperature curves; one at $T^{*} \approx 1$ and the other at $T^{*}$ $\approx 0.4$. As temperature decreases further, the heat-capacity reaches a maximum at $T^{*}=0.18$. Again, this maximum appears to signal a reorganization transition since the radius-ofgyration change is small. The heat-capacity data suggest that the square-well 16mer has three equilibrium transitions. The first transition (starting at high temperature) corresponds to the collapse transition at $T^{*} \approx 1$ which is characterized by a sudden reduction of the polymer chain dimension, while the second and third (at $T^{*} \approx 0.4$ and 0.18 , respectively) are reorganization transitions that allow the chain to reach a lower energy level. Three transitions are also seen for rigidlybonded 12mers (Fig. 3).

\section{4. $32 m e r$}

Figure 8 shows the temperature dependence of the equilibrium properties for an isolated $32 \mathrm{mer}$. The MC and MD results for the heat capacity and internal energy agree with each other very well from $T^{*}=0.45$ to $T^{*}=5$; no MC calculations were made below $T^{*}=0.45$. A $C_{v}$ plateau at $T^{*}$ $\approx 1.3$ signals a collapse transition. As the temperature is lowered further, there is a strong transition at $T^{*} \approx 0.27$. For $T^{*}=0.24-0.27$, different runs with different random initial

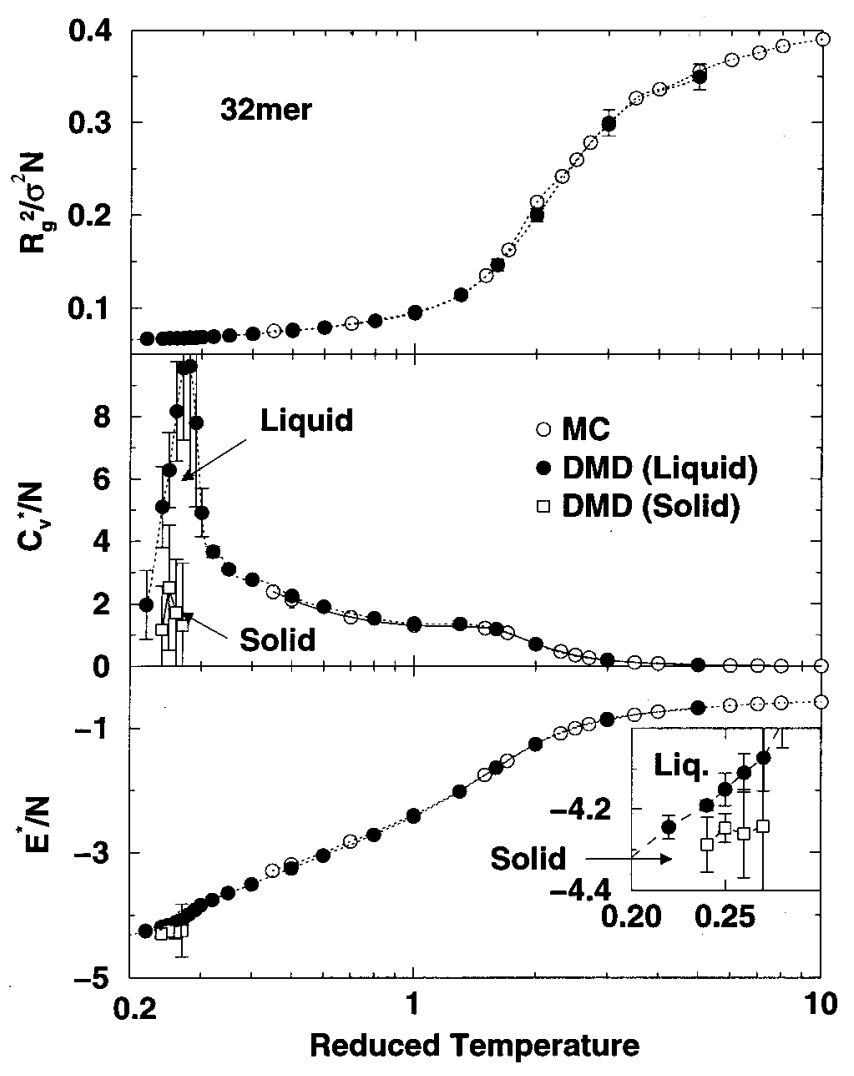

FIG. 8. As in Fig. 6 except for an isolated 32mer. The solid states are identified based on $\Delta_{B}$ values and are averaged over two or three independent runs. (Only two or three out of ten independent runs yield "pure", solid-like states.) The inset shows the details of the low-temperature behavior of the two states in equilibrium.

configurations at the same temperature yield two distinct equilibrium states. In most cases, the two states are stable over 250 million equilibrium collisions while in certain runs, the system oscillates from one state to the other. One of the two states is liquid-like because it has a high internal energy and high $\Delta_{B}(\sim 0.2)$ while the other one is solid-like with a lower internal energy and low $\Delta_{B}(<0.1)$. Thus, we have a two-state liquid-to-solid transition occurring over a wide temperature range, a typical phenomenon for a small finite system (e.g. LJ clusters ${ }^{46}$ ).

\section{5. $64 \mathrm{mer}$}

The equilibrium results for 64mers are shown in Fig. 9. Again, the sigmoidal shape of the $R_{g}^{2}$ vs temperature curve indicates the presence of the well-known collapse transition. The collapse transition is also indicated by a heat-capacity vs temperature peak at $T^{*}=1.5$, a very weak peak (or plateau) in MD results and a stronger peak in MC results. As the temperature decreases further, the squared radius of gyration, which becomes nearly a constant $(\sim 0.05 N)$, gives no indication of any other transitions. However, a first-order transition is evident from the appearance of a "discontinuity" 101 in internal energy at $T^{*} \sim 0.33$. This "discontinuity" $\left[E^{*}\left(T^{*}=0.34\right)-E^{*}\left(T^{*}=0.32\right) \approx 0.5 N\right]$, is accompanied 


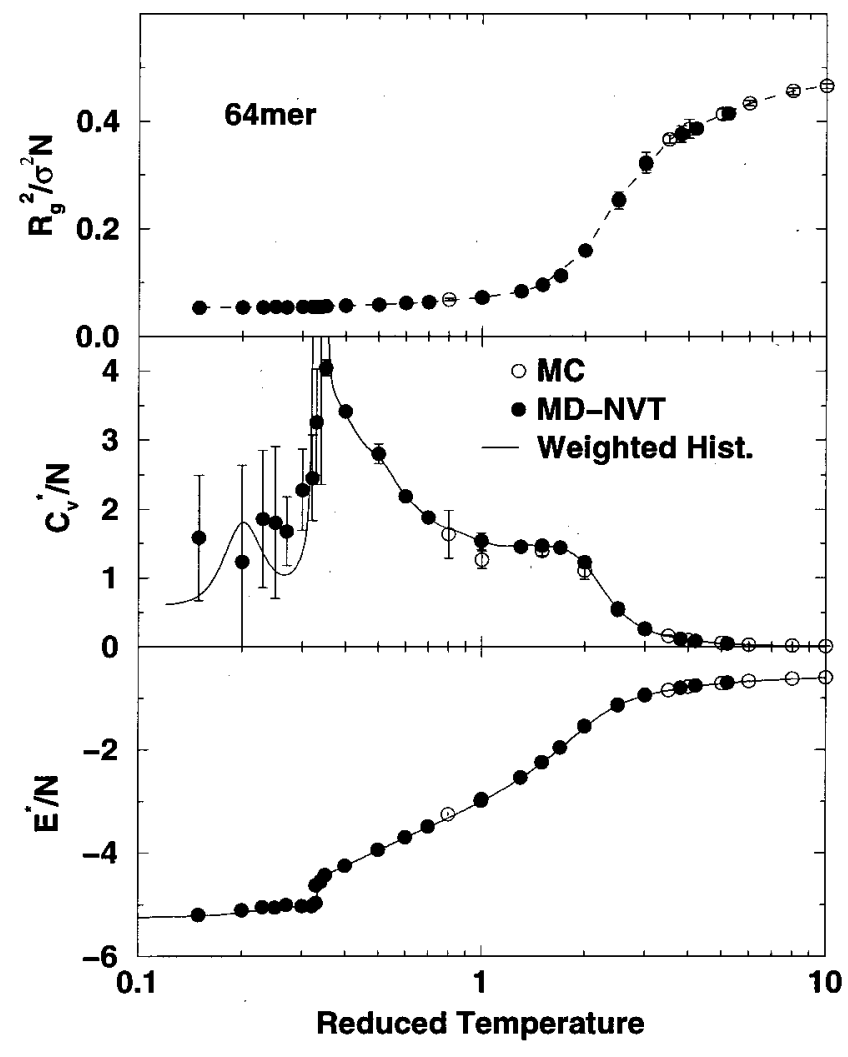

FIG. 9. As in Fig. 6 except for an isolated 64mer. The solid line is obtained from the weighted histogram method (see the Appendix).

by large ("infinite") value of the heat capacity $\left(C_{v}^{*} \sim 30 \mathrm{~N}\right.$ at $T^{*}=0.33$ and 0.34 in certain runs). At lower temperature, there appears to be another transition.

The first-order transition in an isolated 64mer is further verified by the appearance of the free-energy barrier at $T^{*}$ $=0.336$ between two energy levels, which we shall call $A$ $\left(E_{A}=-287 \epsilon\right)$ and $B\left(E_{B}=-318 \epsilon\right)$ (Fig. 10). [The conformational free-energy shown in Fig. 10 is obtained via the weighted histogram method; see Eq. (A5) in the Appendix.] The energy difference between the energies of $A$ and $B, 31 \epsilon$, explains the observed energy "discontinuity" $E^{*}\left(T^{*}\right.$ $=0.34)-E^{*}\left(T^{*}=0.32\right) \approx 0.5 N$. The energy barrier between low-energy state $B$ and transition state $C, E_{C}-E_{B}$, is $17 \epsilon$. Snapshots of the structures for energy levels A and B (Fig. 11) suggest that the latter has a hexagonal-like symmetry while the former is more disordered. Structure $\mathrm{C}$ appears to be somewhat elongated and more disordered than either A or B. The radii of gyration $R_{g}^{2}$ for the energy levels $\mathrm{A}$ and $\mathrm{B}$ are calculated during one simulation at $T^{*}=0.33 . R_{g}^{2} / \sigma^{2}$ for the energy level A is 3.514 (averaging over 5 million configurations) and $R_{g}^{2} / \sigma^{2}$ for the energy level $\mathrm{B}$ is 3.556 (averaging over 18 million configurations); thus, $\left(R_{g}^{2}\right)_{A}-\left(R_{g}^{2}\right)_{B}$ is only $0.04 \sigma^{2}$. This is why the radius of gyration data does not provide strong evidence for the existence of the first-order transition (Fig. 9). In Fig. 12, the instantaneous internal energy is plotted as a function of number of collisions (time) at $T^{*}=0.33$. The figure shows that near the transition tempera-

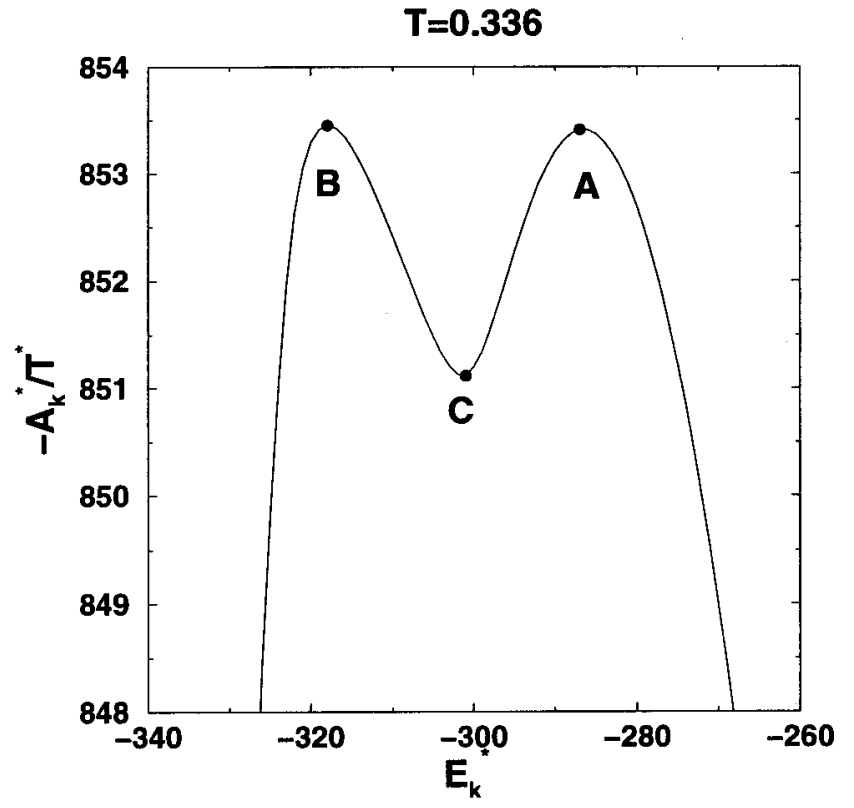

FIG. 10. Reduced conformational free energy $\left(-A_{k}^{*} / T^{*}\right)$ as a function of conformational energy of individual energy state $k$ (obtained via weighted histogram method, see Appendix) at $T^{*}=0.336$. The energy state $B$ $\left(E_{B} / \epsilon=-318\right)$ and the energy state $A\left(E_{A} / \epsilon=-287\right)$ are separated by a free-energy barrier. The energy of the transition state $C, E_{C} / \epsilon$, is -301 .

ture, the chain fluctuates between two stable states that have different average internal energies.

To examine the nature of this first-order transition, we calculate the rms-bead distance fluctuation, $\Delta_{B}$, as a function of temperature (filled circles, Fig. 13). At low temperature, $\Delta_{B}<0.1$, indicating that the homopolymer is in its solid-like phase. As temperature increases, $\Delta_{B}$ increases as a result of stronger thermal fluctuations. Near $T^{*} \approx 0.33, \Delta_{B}$ changes "discontinuously" from 0.06 at $T^{*}=0.32$ to 0.28 at $T^{*}=0.34$. This indicates an order-disorder melting transition. As temperature increases further, $\Delta_{B}$ reaches a maximum at $T^{*} \approx 2$, which is between the high-temperature heatcapacity peak $T^{*}=1.5$ and $T_{\Theta}^{*}(=3)$. The decrease of $\Delta_{B}$ at high temperature suggests that there are less fluctuations in the bead-bead distances for the random-coil states because the stretched chains are constrained by chain connectivity.

We also evaluate the Lindemann disorder parameter for an individual bead $i, \Delta_{L_{i}}$. Figure 14 shows $\Delta_{L_{i}}$ as a function of the distance of bead $i$ from the chain center of mass at $T^{*}=0.30,0.32$ and 0.34 . At $T^{*}=0.34$ (above the transition temperature $T^{*}=0.336$ ), the system is clearly liquid-like with $\Delta_{L_{i}}>0.7$, independent of the distance from the center. Below the transition temperature $T^{*}=0.336$, the interior of the 64 mer has a more-or-less constant $\Delta_{L_{i}}$. The root meansquared average of $\Delta_{L_{i}}, \Delta_{L}$, over the beads that are less than $1.4 \sigma$ away from the center yields a value of 0.075 . Thus, the interior of the 64mer satisfies Lindemann's rule for a solid below the temperature $T^{*}=0.30$. However, $\Delta_{L}$ for beads at a distance more than $1.4 \sigma$ is 0.26 at the same temperature, suggesting that the motion of beads at the surface is liquidlike. For $T^{*}=0.32$, similar behavior to that at $T^{*}=0.30$ is 


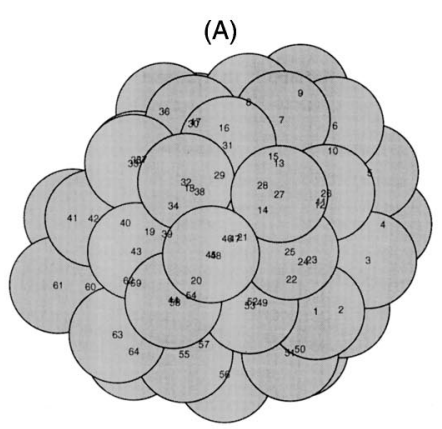

(A)

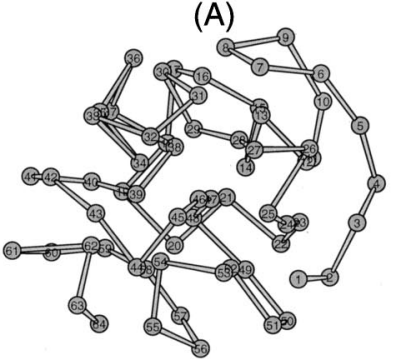

(C)

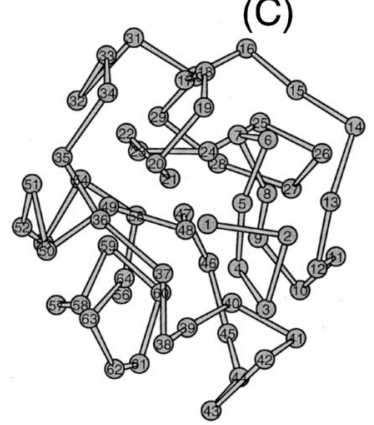

(B)

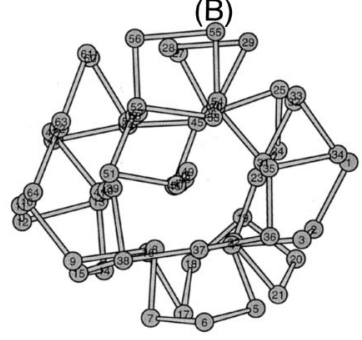

(A)

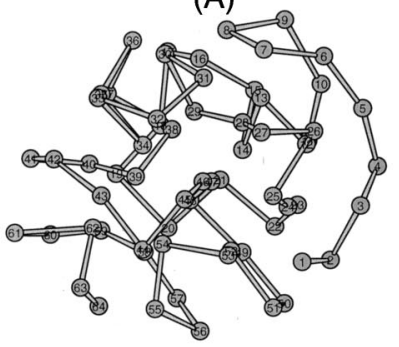

(C)

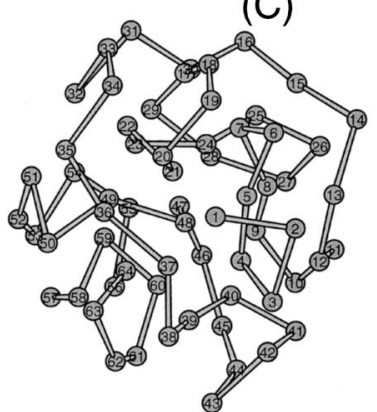

(B)

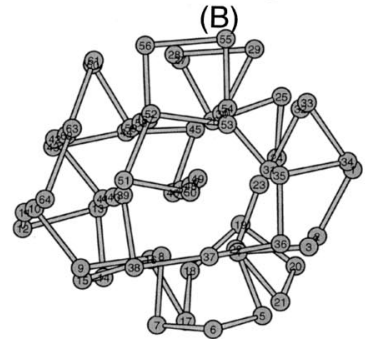

FIG. 11. A snapshot of the structure for energy level A, C and B as labeled. The structure on the top shows the actual packing of the structure A. All other structures are shown in a cross-eyed stereo plot where the bead diameter is smaller than the actual diameter for a clear view.

observed except that $\Delta_{L}$ is somewhat larger for all values of the distance from the center; e.g. for a distance less than $1.4 \sigma, \Delta_{L}=0.12$. This suggests the critical value for Lindemann's rule is $\sim 0.12$ for the liquid-to-solid transition in the interior of the $64 \mathrm{mer}$. This critical value is in good agreement with 0.125 found for a hard-sphere solid. ${ }^{102}$

Figure 15 shows the bead-bead radial distribution function and running coordination number (defined to be the integral of the distribution function over the distance) from five independent runs for an isolated $64 \mathrm{mer}$ at various reduced temperatures. The radial distribution function is nonzero starting from $r / \sigma=1-\delta=0.9$. The bead connectivity produces peaks near $r / \sigma=1,2$, and 3 while the square-well

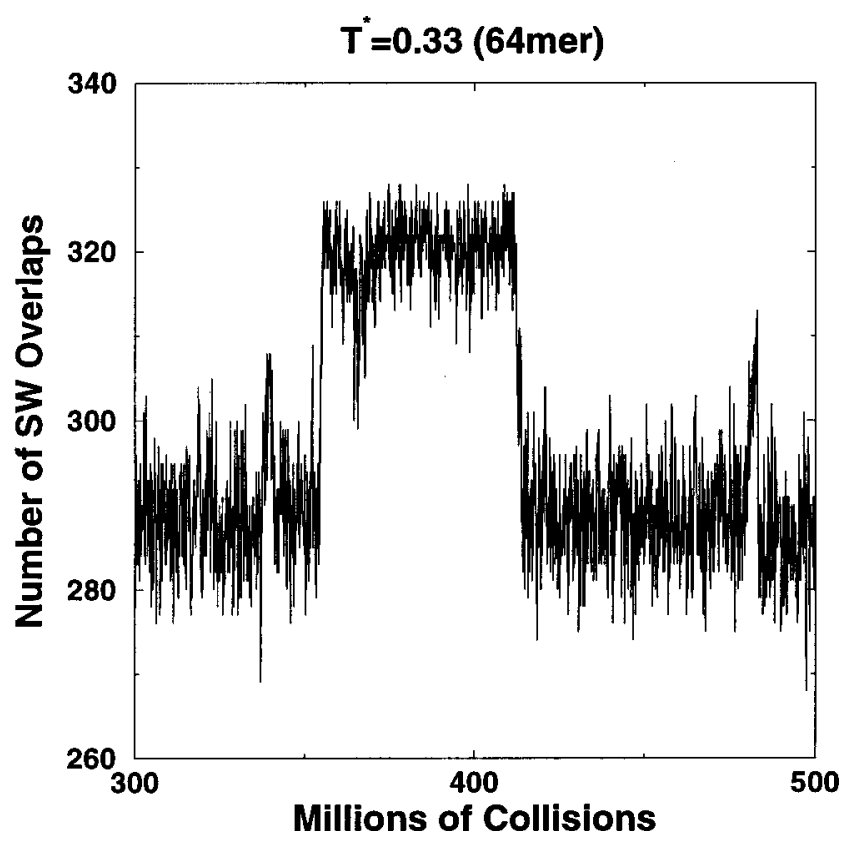

FIG. 12. The instantaneous number of square-well overlaps as a function of time (expressed in term of millions of collisions) for an isolated $64 \mathrm{mer}$ at $T^{*}=0.33$.

potential between nonbonded beads yields a discontinuity at $r / \sigma=1.5$. At $T^{*}=3.80$, the running coordination number is around 2 for the first peak at $r / \sigma$ between 0.9 and 1.1. This simply states the fact that each bead is bonded with its two neighbors. Beyond the first peak, the radial distribution function has only two weak peaks located at $r / \sigma=1.5$ and 2.0 which signal the presence of bead-bead attraction and bead connectivity, respectively. Below the collapse transition

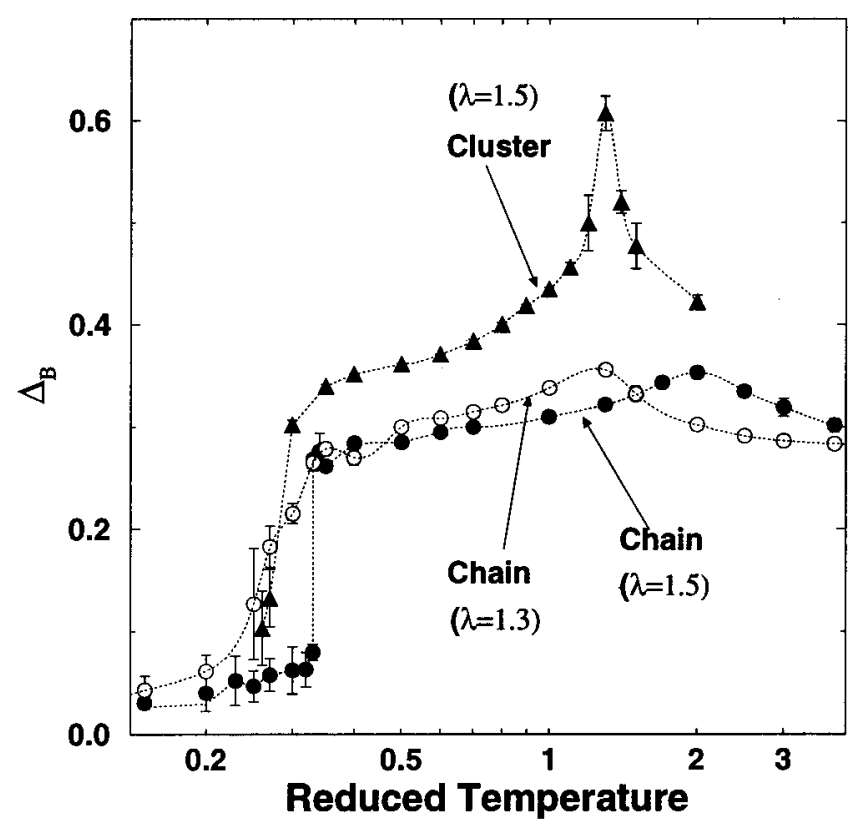

FIG. 13. The relative rms bead-bead distance fluctuation $\Delta_{B}$ as a function of reduced temperature for an isolated square-well 64mer calculated via DMD simulations. Dashed lines serve only as a guide for the eye. 


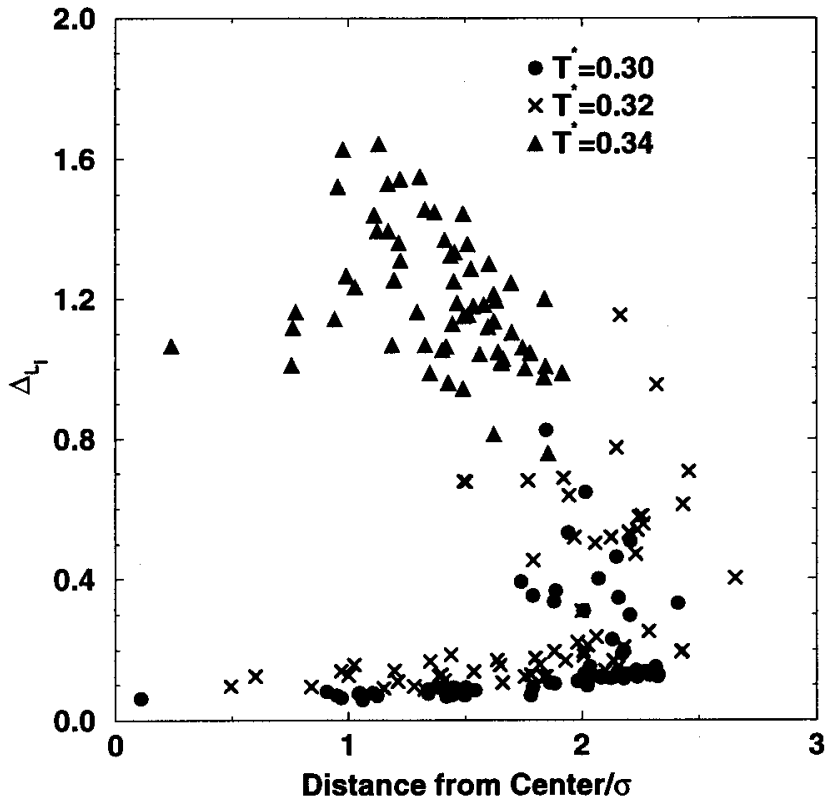

FIG. 14. The individual Lindemann disorder parameter $\Delta_{L_{i}}$ as a function of the distance of bead $i$ from the center of mass.

$\left(T^{*}=1.5\right)$, the overall structures of the radial distribution functions at $T^{*}=1.0$ and 0.35 are similar to the hightemperature $\left(T^{*}=3.8\right)$ structure but the former have more pronounced peaks at $r / \sigma=1.5$ and 2.0. At $T^{*}=0.35$, which is above the liquid-solid transition temperature 0.336 , the number of nearest neighbors (including the bonded beads) reaches 6 . For the solid phases at $T^{*}=0.32$ and 0.20 , the

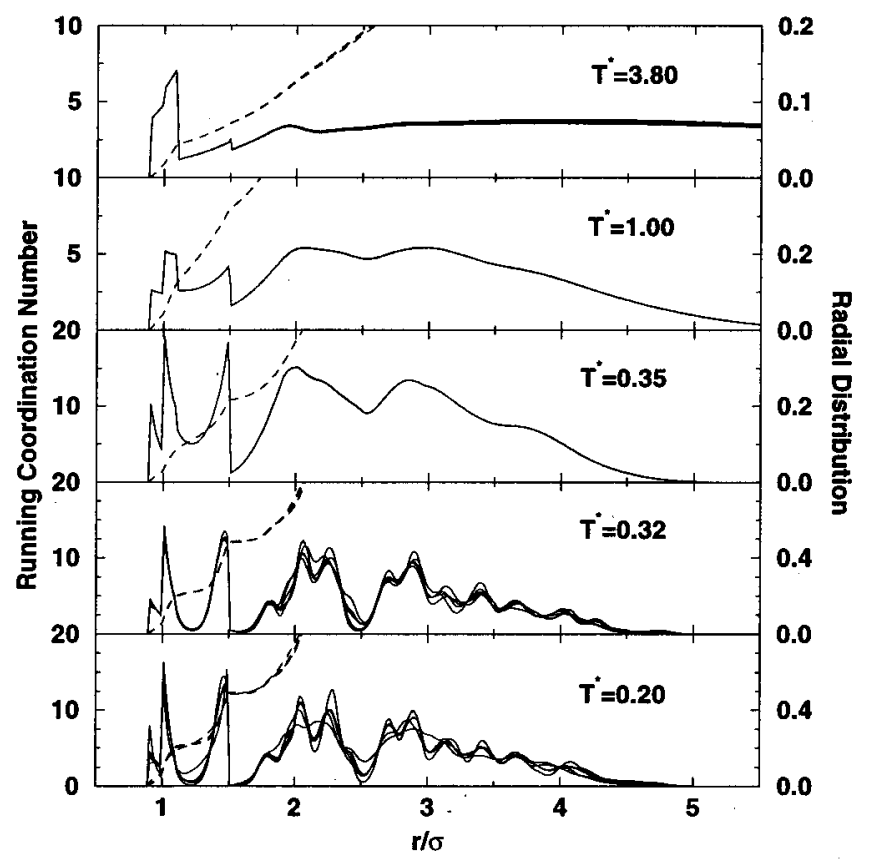

FIG. 15. The radial distribution functions and running coordination numbers obtained from five independent runs at various reduced temperatures for an isolated $64 \mathrm{mer}$. The dashed lines are running coordination numbers.

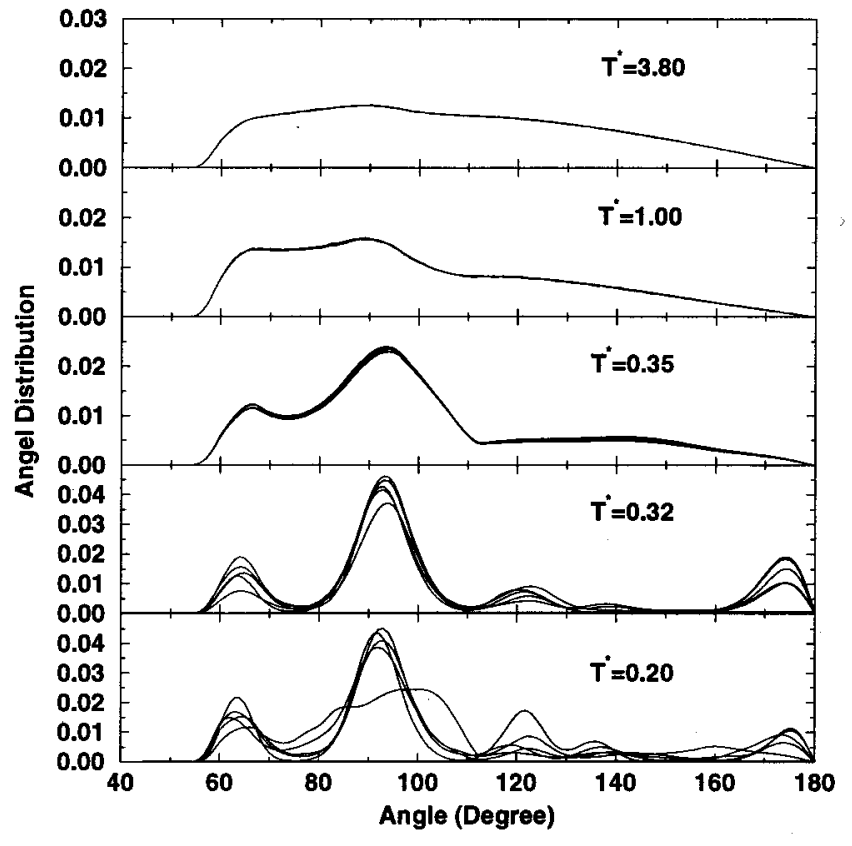

FIG. 16. The bond angle distribution functions obtained from five independent runs at various reduced temperatures for an isolated $64 \mathrm{mer}$.

radial distribution function exhibits many more peaks than those for $T^{*}>0.33$. The multiple peaks in solid structures indicate that the motion of the beads is dominated by the vibrational motion. Based on the values of the running coordination number for solid structures, each bead is surrounded by 6 beads in the first layer $(r / \sigma \sim 1.3)$ and another 6 beads in the second layer $(r / \sigma \sim 1.7)$.

The bond-angle distribution functions at various temperatures are shown in Fig. 16. At high temperature, the distribution is relatively featureless. For structures at $T^{*}$ $=1.0$ and 0.35 (below the collapse transition), there are two peaks at around $65^{\circ}$ and $90^{\circ}$, respectively. This indicates that there is some structure in the liquid-like homopolymer phase. For the solid phases at $T^{*}=0.32$ and 0.20 , additional peaks appear around $120^{\circ}, 135^{\circ}$, and $175^{\circ}$. The first two angles signal hexagon-lattice and square-lattice symmetries, respectively. The near-linear $175^{\circ}$ bond angle also indicates a highly compact lattice structure.

A typical solid structure of a $64 \mathrm{mer}$ at $T^{*}=0.32$ (right below the transition) is shown in Fig. 17. The structure of an isolated 64mer is a rather well-ordered 3-dimensional lattice which mixes hexagonal and cubic lattice symmetry. This mixing occurs because there is an accidental degeneracy for $\lambda=1.5$ with beads arranged in a square having the same energy as beads arranged in a hexagon. As the temperature is decreased below 0.2 , this simple lattice structure undergoes a continuous polymorphic solid-solid transition (Fig. 9) into a more-spherically-shaped structure which has less symmetry but a lower energy (Fig. 18). This polymorphic transition may be categorized as a displacive polymorphic transition, ${ }^{103}$ in which the low-temperature structure is more ordered (with a lower entropy) but has lower symmetry than the hightemperature structure. 

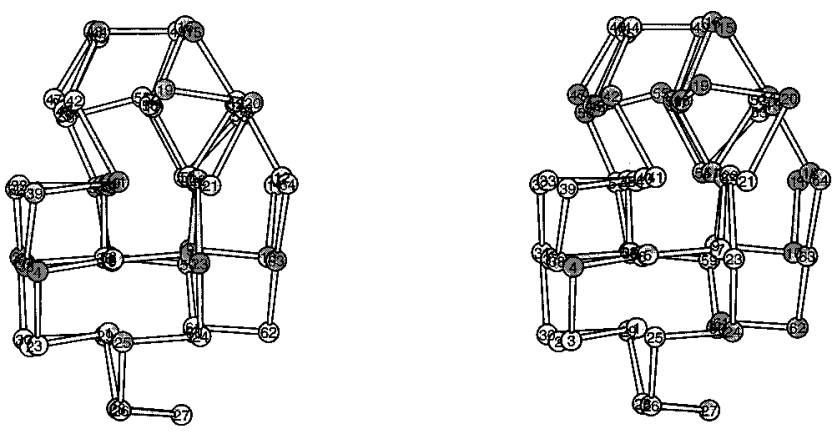

FIG. 17. A cross-eyed stereo plot of a typical snapshot of the $64 \mathrm{mer}$ at $T^{*}=0.32$.

The chain-length dependence of the simulation results can be summarized as follows. As the chain length increases, the number of transitions increases from one for the $3 \mathrm{mer}$, $4 \mathrm{mer}$ and $5 \mathrm{mer}$, to two for the $6 \mathrm{mer}, 7 \mathrm{mer}$, and $8 \mathrm{mer}$, and to three for the $12 \mathrm{mer}, 16 \mathrm{mer}$, and $64 \mathrm{mer}$. Although the $32 \mathrm{mer}$ only exhibits two transitions, a third transition could occur at $T^{*}<0.24$, the lowest temperature investigated here. As chain length increases from 32 to 64 , the temperature at which the liquid-to-solid transition occurs increases, along with the strength of the transition. Meanwhile, the temperature range over which two states coexist becomes narrower, in accord with the fact that the temperature range is expected to approach zero for a chain composed of an infinite number of particles.

\section{DMD results for $\lambda=1.3(64 \mathrm{mer})$}

It is of interest to know how a change in the square-well diameter affects the transition behavior of a square-well chain. In Figure 19, the DMD results for radius of gyration, heat-capacity, and internal energy of an isolated 64mer at $\lambda$ $=1.3$ are compared with those at $\lambda=1.5$. As the figure shows, the internal energy at $\lambda=1.3$ is always higher than that at $\lambda=1.5$ because of the lower overall attraction in the former model. As has been observed for short chains, ${ }^{15}$ a shorter-ranged attraction leads to a stronger gas-to-liquidlike collapse transition at a lower temperature. However, it is somewhat surprising that the low-temperature transition weakens from a bimodal energy distribution (first-order-like; see Fig. 10) at $\lambda=1.5$ to a unimodal distribution at $\lambda=1.3$
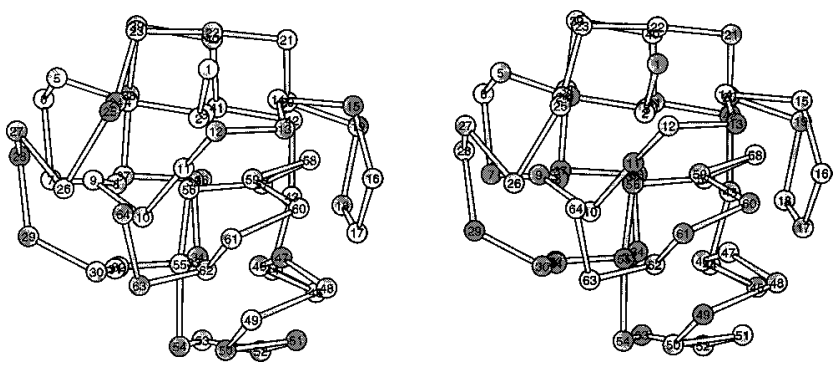

FIG. 18. A cross-eyed stereo plot of the structure of a low energy state $\left(E^{*}=-342\right)$ found in 1 billion collisions of a simulation annealing from $T^{*}=1.0$ to $T^{*}=0.05$.

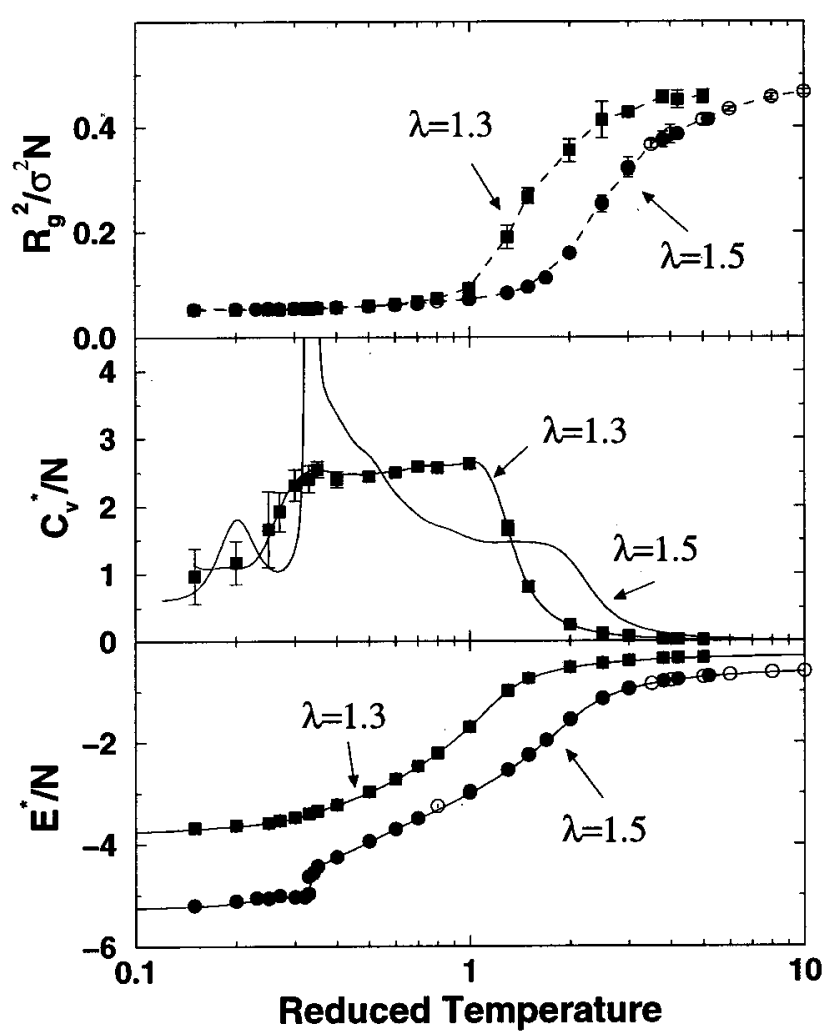

FIG. 19. The DMD thermodynamic results for $\lambda=1.3$ are compared with the results for $\lambda=1.5$.

(not shown). A weaker low-temperature transition is also reflected in the rms bead-bead distance fluctuation $\Delta_{B}$ curve (open circles) shown in Fig. 13; the quantity $\Delta_{B}$ gradually passes the critical value of 0.1 at $T^{*}<0.25$ rather than suddenly, as is the case of $\lambda=1.5$ at $T^{*} \sim 0.33$. Despite the significant difference in the transition strength at low temperatures, the values of the radii of gyration for $\lambda=1.5$ and 1.3 are nearly the same around $T^{*}=0.35$. This suggests that the range of attraction (not just packing) is crucial for a strong transition to a solid in a finite system. The relativelyfixed liquid-to-solid transition temperature and the decrease of the gas-to-liquid transition temperature with decreasing $\lambda$ suggest that the two transitions might combine into one if the square-well diameter were to decrease further.

To see if there is a difference between the lowtemperature solid-like structures of the 64 mer with $\lambda=1.5$ and those of the 64 mer with $\lambda=1.3$, the comparison between the radial distribution and angle distribution functions at $T^{*}=0.2$ is made in Fig. 20. The number of peaks in the radial distribution for $\lambda=1.3$ is less than the distribution function for $\lambda=1.5$. This suggests that at $T^{*}=0.2$, the 64 mer for $\lambda=1.3$ is less "solid"'-like than the 64mer with $\lambda=1.5$, even though $\Delta_{B}<0.1$ for both cases. The running coordination for $\lambda=1.3$ is 5 for the first and second peaks, which is smaller than 6 for $\lambda=1.5$. The angle distribution further shows significant structural differences. The bond angles of the 64 mer with $\lambda=1.5$ have a large probability to be at $90^{\circ}$ while the probability of the 64 mer with $\lambda=1.3$ 

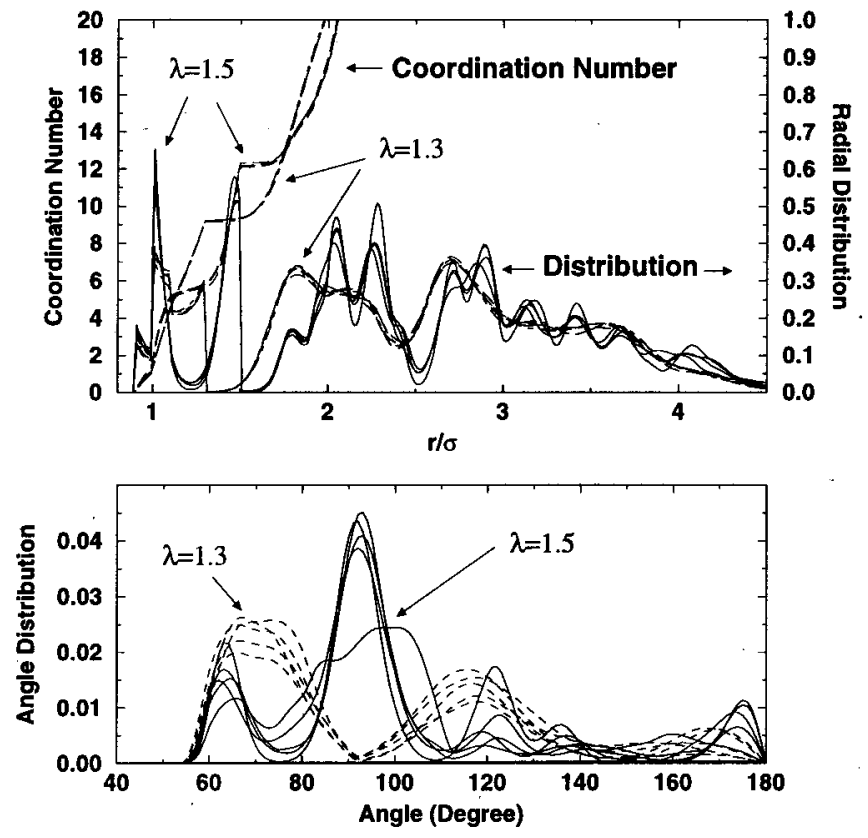

FIG. 20. The radial distribution and bond angle distribution functions from five independent runs at $T^{*}=0.2$ for the cases $\lambda=1.3$ and 1.5 as labeled.

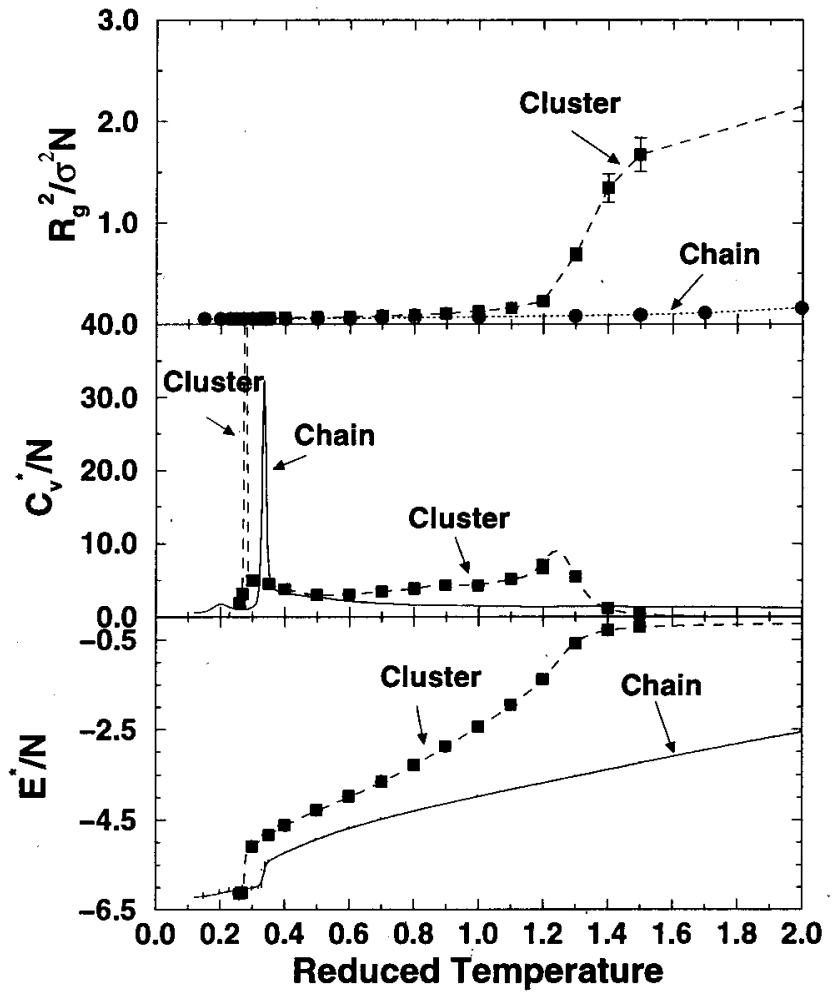

FIG. 21. The DMD results for the thermodynamics of a square-well cluster of 64 beads are compared with those results for a square-well chain of 64 beads. The lines (except for the radius of gyration) are obtained via the weighted histogram method (see Appendix). The lines for the radius of gyration serve only as a guide for the eye. The internal energy of the chain here also includes the neighboring bond-energy $(-63 \epsilon)$ to facilitate comparison.

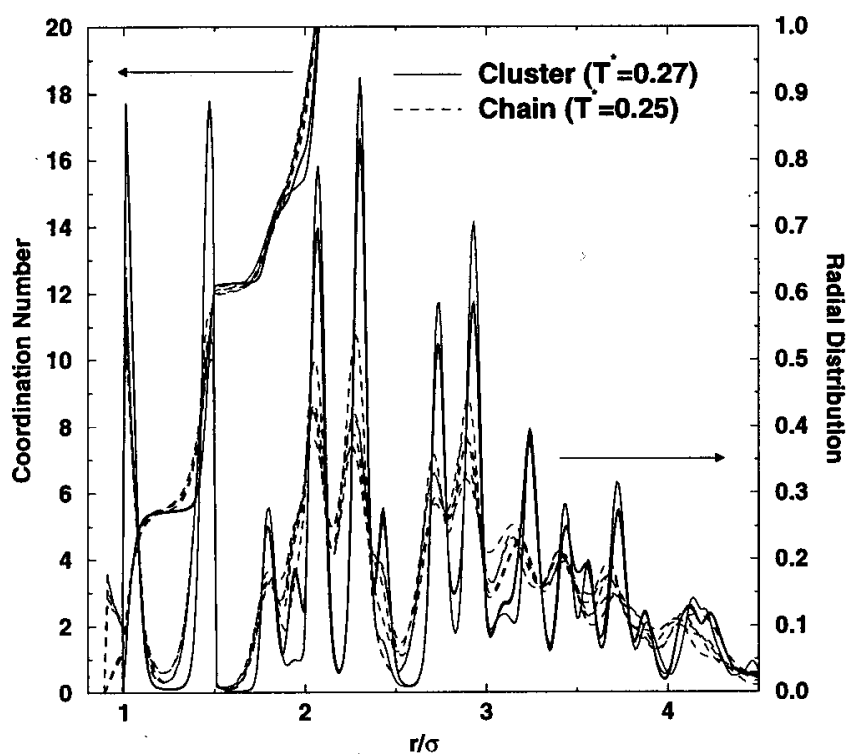

FIG. 22. The radial distribution functions of cluster and chain obtained from five independent runs.

having a $90^{\circ}$ bond angle is nearly zero. This is because $90^{\circ}$ bond-angle yields a square-well overlap when $\lambda=1.5$ but not when $\lambda=1.3$.

\section{Square-well cluster of 64 square-well beads}

The mean-squared radius of gyration, the heat-capacity and internal energy for a square-well cluster composed of 64 beads are compared in Fig. 21 with results for a $64 \mathrm{mer}$ square-well chain having the same square-well diameter $\lambda$ $=1.5$. The chain and the cluster have similar values of the radius of gyration at temperatures below $T^{*}=0.8$. For $T^{*}$ $>0.8$, both models experience a sudden increase in the radius but the increase in the cluster size is much stronger since the cluster experiences no bond constraints. (The sudden increase in $R_{g}^{2}$ for chains is not visible in this figure because of the scale; readers are referred to Fig. 9 instead.) The absence of "bond" constraints in a cluster also leads to stronger gas-liquid and liquid-solid transitions at lower transition temperatures. From the chain to the cluster, the gasliquid-like transition temperature decreases from $T^{*}=1.5$ to $T^{*}=1.25$ and the liquid-solid transition temperature decreases from 0.336 to 0.28 . Since there are fewer constraints, a lower temperature is required to induce transitions. The overall transition patterns of an isolated cluster and chain are similar even though the bond constraint in the chain significantly weakens the gas-to-liquid like collapse transition.

The solid structure of the cluster is remarkably similar to that of a SW chain. Figure 22 shows that the running coordination numbers for the two systems are the same and the radial distribution of the cluster is nearly identical to that of a chain except that the peaks for the cluster are sharper.

The thermodynamic results for the SW cluster are qualitatively similar to those for the LJ cluster. Both exhibit two 
transitions that correspond to gas-to-liquid and liquid-tosolid transitions for the bulk phase ${ }^{48}$ and both liquid-to-solidlike transitions are first order. ${ }^{49}$

\section{DISCUSSION}

The present results show that an isolated square-well homopolymer chain exhibits multiple equilibrium transitions similar to SW and LJ clusters. In contrast, most previous homopolymer simulations $1,2,4-6,8-10,13,14,19,104-115,99,100,116-120$ (except those for relatively esoteric lattice chain models ${ }^{17,38,39}$ ) have only found a single transition. This can be explained as follows. The majority of the previous

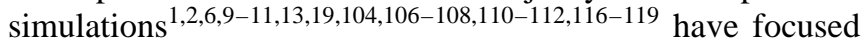
primarily on the chain dimensional quantities, such as the radius of gyration and end-to-end distance. As demonstrated here, they are not good indicators of a liquid-solid-like and/or a polymorphic solid-solid transition since the variation in the radius of gyration for these transitions is very small (e.g., the difference in $R_{g}^{2}$ is $\sim 0.04 \sigma^{2}$ for the liquidto-solid transition of 64mer). Further, there is a lack of heatcapacity data, which are a sensitive indicator of different transitions, due mainly to the difficulty associated with obtaining converged results. ${ }^{48}$ Most of the existing heat capacity calculations deal with lattice models..$^{8,105,113,115}$ As we have shown, decreasing the range of the attractive interaction (i.e., the well width) weakens the liquid-solid-like transition. Thus, nearest neighbor interactions in lattice models, which have a well width of zero (or, equivalently, $\lambda=1$ ), may have weakened the liquid-to-solid transition so much that it is masked by the collapse transition. In an off-lattice MC simulation of heat-capacities as a function of temperature, Milchev et $a .^{5}$ used a Morse potential whose attraction range is short $(\lambda \approx 1.25)$ and did not find a liquid-to-solid transition. Karasawa and Goddard III ${ }^{120}$ calculated the heatcapacity for isolated polymethylene; however, their results are not accurate enough to determine if there is multitransition behavior. Baumgärtner obtained heat-capacities for isolated LJ chains ${ }^{99,100}$ which indicated that there was only one transition. He suggested that this transition is a liquidsolid-like transition because the temperature at which the heat-capacity exhibited a maximum was much smaller than the $\Theta$-temperature $\left(T^{*} \approx 3.7\right)$ and because the change in the radius of gyration was very small. His suggestion is in accord with our findings. It is interesting to note that his heat capacity results did not show any trace of a collapse transition. This may be caused by the fact that the liquid-to-solid transition masked the collapse transition. Recently, López found that the J-walking Monte Carlo method yielded a firstorder liquid-to-solid like transition with a bimodal distribution of energy for a cluster of $55 \mathrm{LJ}$ atoms while the standard Metropolis MC method generated a weaker transition with a unimodal distribution of energy. ${ }^{49}$ Thus, it is unclear whether the transition found in $\mathrm{LJ}$ chains would be changed by use of a different MC algorithm.

The existence of complex-phase behavior for isolated homopolymer chains is supported by several recent lightscattering experiments on poly $(\mathrm{N}$-isopropylacrylamide $)$ that indicate the existence of long-lived kinetic ${ }^{26}$ and equilibrium intermediates. $^{28,29}$ Microcalorimetric measurements of poly $(\mathrm{N}$-isopropylacrylamide $)$ and poly $(\mathrm{N}$-isopropylmethacrylamide) also show that these homopolymer transitions can have the "two-state" and "domain" character of cooperative transitions. ${ }^{25}$ More recently, a solid-to-solid polymorphic transition was predicted ${ }^{121}$ for particles interacting with square-well or square-shoulder potentials in two dimensions and observed ${ }^{122}$ in a confined colloid suspension of uncharged polymethylmethacrylate spheres that exhibited firstorder liquid-to-hexatic and hexatic-to-"solid" transitions. This suggests that the solid-solid polymorphic transition found here for a SW homopolymer is real. Our recent simulations of heteronuclear 46mers further suggest that the solid-solid polymorphic transition in the interior of the collapsed globule coincides with the liquid-to-solid transition of the whole chain based on Lindemann's rule. ${ }^{123}$ It is to be noted that a corresponding phase complexity is found for a cluster of $55 \mathrm{LJ}$ atoms which coexist in three phases: liquid, surface-melted solid, and solid at certain temperatures. ${ }^{47}$

One question about the complex homopolymer phase diagram presented here is whether it is caused by the short range and discontinuous feature of the square-well potential. We cannot give a direct answer because the DMD simulations are required to obtain well converged results for the thermodynamics. However, the physical origins of all the transitions have been identified; they are a gas-to-liquid-like collapse and a liquid-to-solid transitions of the chain interior (Fig. 14) for the first two transitions at high temperatures and, possibly, a freezing transition of the liquid surface ${ }^{123}$ for the low-temperature "polymorphic" transition. In other words, there is no "strange" or unphysical transitions due to the square-well potentials. It is known that the critical phenomena predicted for a fluid composed of particles interacting with a square-well potential ${ }^{124}$ are as realistic as those predicted for a fluid composed of particles interacting with a LJ potential. Moreover, we have shown that the phase behavior of SW clusters is isomorphic with that of $\mathrm{LJ}$ clusters. $^{48,47}$ Furthermore, the heat-capacity peak for LJ chains was identified as the liquid-to-solid transition. ${ }^{99,100}$ In other words, a LJ chain has at least two transitions: the collapse and liquid-to-solid transitions even though the former is not strong enough to produce a heat-capacity peak. Thus, it is likely that the phase behavior observed for SW chains is physically meaningful.

To observe a liquid-to-solid transition for an isolated homopolymer in an experiment, the transition temperature should be around room temperature $(T \sim 300 \mathrm{~K})$. For a 64mer, this requires a square well depth, $\epsilon$ $\left[=k_{B}\left(T / T^{*}\right)_{\text {trans }}\right]$, to be equal to $1.8 \mathrm{kcal} / \mathrm{mol}$, which is nearly 20 times stronger than $0.1 \mathrm{kcal} / \mathrm{mol}$, the typical well depth for the interaction between methylene groups in vacuum [CHARMM (Ref. 125) parameter set 19 (Ref. 126)]. Such a large well-depth would require the use of a homopolymer composed of different monomers, perhaps involving aromatic groups. For a collapse transition at $T_{\Theta}$ $=300 \mathrm{~K}$, however, $\epsilon$ is only required to be $0.2 \mathrm{kcal} / \mathrm{mol}$. Such an interaction strength can be easily achieved by dis- 
solving the homopolymer in an unfavorable solvent. This explains why the polymer collapse transition is easier to observe than a polymer disorder-to-order transition.

While it may be difficult to show experimentally that multiple equilibrium states in homopolymers exist, proteins have been found to have a complex phase diagram with denatured states, disordered molten globule states, and unique native states. ${ }^{127}$ That a corresponding thermodynamic complexity is found in homopolymer simulations may suggest that multiple states are an essential property of many macromolecules.

Note added in proof. It is interesting to note that Nelson et al. ${ }^{129}$ have recently made phenylacetylene oligomers that form stable helices involving strong stacking interactions between the aromatic groups. This supports our suggestion that homopolymers involving aromatic interactions could have liquid to solid transitions at room temperature.

\section{ACKNOWLEDGMENTS}

We would like to thank Dr. R. S. Berry, Dr. C. L. Brooks, Dr. M. Taylor, Dr. S. Smith, Mr. P. Gupta, and Mr. H. Gulati for helpful discussions and/or comments. The work at Harvard was supported by the National Science Foundation. The work at NCSU was supported by the Director, Office of Energy Research, Basic Sciences, Chemical Sciences Division of the U. S. Department of Energy and by the National Science Foundation under Grant No. CTS-9208590. We also thank the Donors of the Petroleum Research Fund administered by the American Chemical Society for partial support of this work and the North Carolina Supercomputing Center for $200 \mathrm{~h}$ time on their Cray Y-MP and T-90 supercomputers. This material is based in part on work supported by a postdoctoral fellowship (Y.Z.) from the Program in Mathematics and Molecular Biology at the University of Berkeley, which is supported by the National Science Foundation under Grant No. DMS-9406348. The Government has certain rights in this material. The calculations at Harvard were conducted on HP 9000/700 workstations and on DEC Alpha workstations. The structures of the chains are drawn by ASGL, a program developed by A. Sali.

\section{APPENDIX: THE WEIGHTED HISTOGRAM METHOD FOR DMD SIMULATIONS}

Consider a system which has $K$ energy levels each with energy $E_{k}, k=1,2, \ldots, K$. The probability (normalized population) of state $k$ at a given simulation temperature $T_{l}$, $P_{k}\left(T_{l}\right)$, can be obtained from DMD simulations,

$$
P_{k}\left(T_{l}\right)=\frac{t_{k}\left(T_{l}\right)}{t_{\mathrm{eq}}\left(T_{l}\right)},
$$

where $t_{k}\left(T_{l}\right)$ is the total time during which the system is in the energy state $k$ and $t_{\mathrm{eq}}\left(T_{l}\right)$ is the total equilibrium simulation time at temperature $T_{l}$. Obviously, one has $\sum_{k=1}^{K} P_{k}\left(T_{l}\right)=1$.
The weighted histogram method is a least-square optimization method to extract the degeneracy factors $g_{k}$ from simulations at several temperatures $\left(T_{l}, l=1,2, \ldots, L\right)$ from the formally exact expression

$$
g_{k}=\frac{\sum_{l=1}^{L} P_{k}\left(T_{l}\right)}{\sum_{l=1}^{L} e^{-\beta_{l} E_{k}-f_{l}}},
$$

where $\beta_{l}=1 / k_{B} T_{l}$ and $f_{l}$ is an optimization parameter, which is related to the Helmholtz free energy at temperature $T_{l}$ via $f_{l}=-\beta_{l} A\left(T_{l}\right)$. This equation is a slightly modified version of the original Ferrenberg-Swendsen equation: ${ }^{52}$

$$
g_{k}=\frac{\sum_{l=1}^{L} t_{k}\left(T_{l}\right)}{\sum_{l=1}^{L} t_{\mathrm{eq}}\left(T_{l}\right) e^{-\beta_{l} E_{k}-f_{l}}} .
$$

Equation (A3) is equivalent to Eq. (A2) if the simulation length $t_{\mathrm{eq}}\left(T_{l}\right)$ is the same for all temperatures. Equation (A2) is used in this paper since it gives the same weight to the long simulations at low temperature and the short simulations at high temperature. The unknown parameter $f_{l}$ in Eq. (A2) can be obtained self-consistently from the equation for the free energy,

$$
f_{l}=-\beta_{l} A\left(T_{l}\right)=\ln \left(\sum_{k=1}^{K} g_{k} e^{-\beta_{l} E_{k}}\right) .
$$

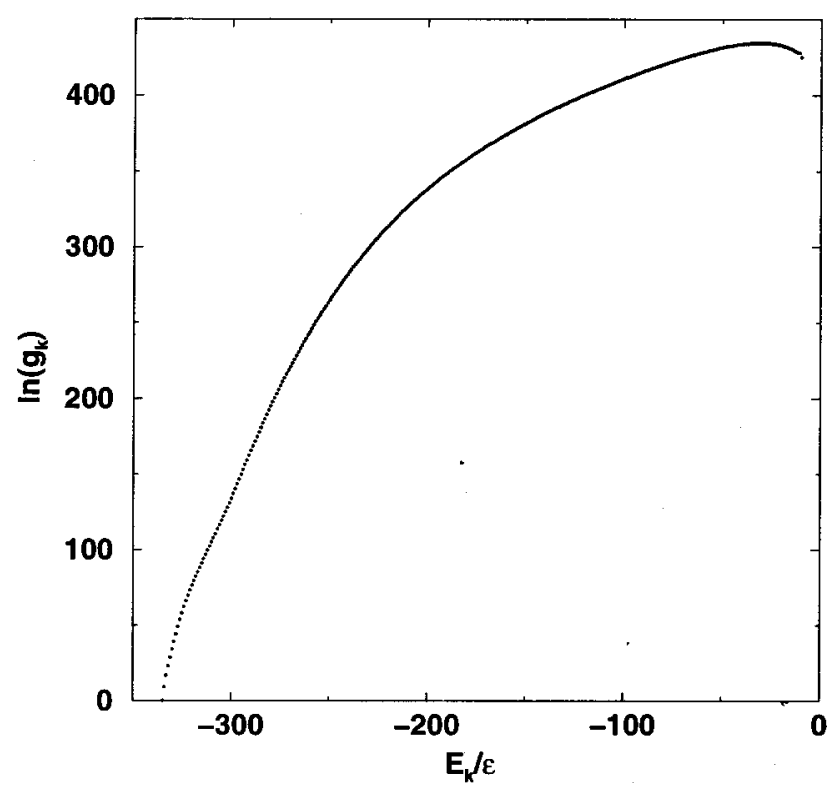

FIG. 23. The logarithm of the degeneracy factor $g_{k}$ (normalized by $g_{k}$ at $\left.E_{k}=-335\right)$ as a function of the reduced energy level $E_{k} / \epsilon$ for a 64 mer with $\lambda=1.5$. Each dot corresponds to a discrete energy level. The increment from one energy level to the nearest higher level is always $\epsilon$. The normalization used here is only for the purpose of convenience since only the relative values for $g_{k}$ can be obtained from the weighted histogram method. 
Once $f_{l}$ is known, the degeneracy factor $g_{k}$ can be calculated from Eq. (A2). Then, the partition function at any temperature can be calculated from Eq. (12) and all other thermodynamic properties can be obtained from Eqs. (13), (14) and (15). To determine $f_{l}$ and $g_{k}$, Eqs. (A2) and (A4) are solved iteratively with energy-population simulation data $P_{k}\left(T_{l}\right)$ as input. The detailed procedure is as follows. First, an initial guess for $f_{l}$ is made ( $f_{l}=0$ is used) and $g_{k}$ is calculated from Eq. (A2). Then, a new improved guess for $f_{l}$ is obtained from Eq. (A4). The iteration continues until the differences between $f_{l}$ values from two successive iterations are less than $0.01 \%$. It should be noted that only relative values of $g_{k}$ are obtained since $g_{k}$ multiplied by any constant can satisfy both Eqs. (A2) and (A4).

To facilitate the comparison between different energy states, one defines the conformational free energy for energy level $k$,

$$
\beta A_{k}^{\text {conf }}=-\ln \left(g_{k} e^{-\beta E_{k}}\right)=\beta E_{k}-\ln g_{k} .
$$

Since $A=E-T S$, one can also define the conformational entropy for the individual energy level $k$ as

$$
S_{k}^{\mathrm{conf}}=k_{B} \ln g_{k} .
$$

Investigation of $A_{k}^{\text {conf }}$ and $S_{k}^{\text {conf }}$ as a function $E_{k}$ is one of the methods for determining if there is a free energy barrier between energy levels. ${ }^{128}$ Figure 23 shows the logarithm of the degeneracy factor $g_{k}$ as a function of $E_{k}$ for a 64mer with $\lambda=1.5$. The concave-upward shape of the $\ln g_{k}$ curve around $E_{k}=-300 \epsilon$ indicates a first-order transition; ${ }^{128}$ i.e., such a $\ln g_{k}$ dependence combined with $-\beta E_{k}$ yields a barrier in the free energy [Eq. (A5) and Fig. 10].

${ }^{1}$ I. Szleifer, E. M. O'Toole, and A. Z. Panagiotopoulos, J. Chem. Phys. 92, 6802 (1992).

${ }^{2}$ A. Rey, J. J. Freire, M. Bishop, and J. Clarke, Macromolecules 25, 1311 (1992).

${ }^{3}$ H. S. Chan and K. A. Dill, J. Chem. Phys. 99, 2116 (1993).

${ }^{4}$ J. M. Victor and J. B. Imbert, Europhys. Lett. 24, 189 (1993).

${ }^{5}$ A. Milchev, W. Paul, and K. Binder, J. Chem. Phys. 99, 4786 (1993).

${ }^{6}$ A. Sikorski, Polymer 34, 1271 (1993).

${ }^{7}$ H. H. Gan and B. C. Yu, J. Chem. Phys. 100, 5922 (1994).

${ }^{8}$ P. Grassberger and R. Hegger, J. Chem. Phys. 102, 6881 (1995).

${ }^{9}$ A. M. Rubio, J. J. Freire, J. H. R. Clarke, C. W. Yong, and M. Bishop, J. Chem. Phys. 102, 2277 (1995).

${ }^{10}$ A. M. Rubio, J. J. Freire, M. Bishop, and J. H. R. Clarke, Macromolecules 28, 2240 (1995)

${ }^{11}$ G. Tanaka and W. L. Mattice, Macromolecules 28, 1049 (1995).

${ }^{12}$ A. Byrne, P. Kiernan, D. Green, and K. A. Dawson, J. Chem. Phys. 102, 573 (1995)

${ }^{13}$ J. Ma, J. E. Straub, and E. I. Shakhnovich, J. Chem. Phys. 103, 2615 (1995).

${ }^{14}$ Y. A. Kuznetsov, E. G. Timoshenko, and K. A. Dawson, J. Chem. Phys. 103, 4807 (1995).

${ }^{15}$ M. P. Taylor, Mol. Phys. 86, 73 (1995).

${ }^{16}$ M. P. Taylor and J. E. G. Lipson, J. Chem. Phys. 104, 4835 (1996).

${ }^{17}$ Y. A. Kuznetsov, E. Timoshenko, and K. A. Dawson, J. Chem. Phys. 104, 336 (1996).

${ }^{18}$ Y. A. Kuznetsov, E. Timoshenko, and K. A. Dawson, J. Chem. Phys. 104, 3338 (1996)

${ }^{19}$ M. Wittkop, S. Kreitmeier, and D. Göritz, J. Chem. Phys. 104, 3373 (1996).

${ }^{20}$ G. Luna-Bárcenas, G. E. Bennett, I. C. Sanchez, and K. P. Johnston, J. Chem. Phys. 104, 9971 (1996).

${ }^{21}$ S. Doniach, T. Garel, and H. Orland, J. Chem. Phys. 105, 1601 (1996).
${ }^{22}$ B. Chu, I. H. Park, Q. W. Wang, and C. Wu, Macromolecules 20, 2833 (1987).

${ }^{23}$ K. Kubota, S. Fujishige, and I. Ando, J. Phys. Chem. 94, 5154 (1990).

${ }^{24}$ E. I. Tiktopulo, V. E. Bychkova, J. Ricka, and O. B. Ptitsyn, Macromolecules 27, 2879 (1994)

${ }^{25}$ E. I. Tiktopulo, V. N. Uversky, V. B. Lushchik, S. I. Klenin, V. E. Bychkova, and O. B. Ptitsyn, Macromolecules 28, 7519 (1995).

${ }^{26}$ B. Chu, Q. Ying, and A. Y. Grosberg, Macromolecules 28, 180 (1995).

${ }^{27}$ M. Nakata, Phys. Rev. E 51, 5770 (1995).

${ }^{28} \mathrm{C}$. Wu and S. Zhou, Macromolecules 28, 8381 (1995).

${ }^{29}$ C. Wu and S. Zhou, Phys. Rev. Lett. 77, 3053 (1996).

${ }^{30}$ P. G. de Gennes, J. Phys. (France) Lett. 36, L55 (1975).

${ }^{31}$ C. B. Post and B. H. Zimm, Biopolymers 18, 1487 (1979).

${ }^{32}$ A. Y. Grosberg and D. V. Kwznetsov, Macromolecules 25, 1970 (1992).

${ }^{33}$ M. A. Moore, J. Phys. A 10, 305 (1977).

${ }^{34}$ I. C. Sanchez, Macromolecules 12, 980 (1979).

${ }^{35}$ Y. Zhou, C. K. Hall, and M. Karplus, Phys. Rev. Lett. 77, 2822 (1996).

${ }^{36}$ I. M. Lifshitz, A. Y. Grosberg, and A. R. Khokhlov, Rev. Mod. Phys. 50, 683 (1978).

${ }^{37}$ R. Finsy, M. Janssens, and A. Bellemans, J. Phys. A 8, L106 (1975).

${ }^{38}$ E. Orlandini, F. Seno, A. L. Stella, and M. Tesi, Phys. Rev. Lett. 68, 488 (1992).

${ }^{39}$ R. Bradley, Phys. Rev. E 48, R4195 (1993).

${ }^{40}$ B. J. Alder and T. E. Wainwright, J. Chem. Phys. 31, 459 (1959).

${ }^{41}$ W. W. Wood, Fundamental Problems in Statistical Mechanics III (NorthHolland/American Elsevier, Amsterdam, New York, 1975), p. 331.

${ }^{42}$ D. C. Rapaport, J. Comput. Phys. 34, 184 (1980).

${ }^{43}$ J. Liu, T. L. Bowman II, and J. R. Elliott, Jr., Ind. Eng. Chem. Res. 33, 957 (1994).

${ }^{44}$ S. W. Smith, C. K. Hall, and B. D. Freeman, Phys. Rev. Lett. 75, 1316 (1995).

${ }^{45}$ S. W. Smith, C. K. Hall, and B. D. Freeman, J. Chem. Phys. 104, 5616 (1996).

${ }^{46}$ R. S. Berry, T. L. Beck, H. L. Davis, and J. Jellinek, Adv. Chem. Phys. 70B, 75 (1988).

${ }^{47}$ R. E. Kunz and R. S. Berry, Phys. Rev. Lett. 71, 3987 (1993).

${ }^{48}$ D. D. Frantz, J. Chem. Phys. 102, 3747 (1995).

${ }^{49}$ G. E. Lopez, J. Chem. Phys. 104, $6650(1996)$.

${ }^{50}$ D. S. Gaunt and S. Flesia, Physica A 168, 602 (1990).

${ }^{51}$ D. S. Gaunt and S. Flesia, J. Phys. A 24, 3655 (1991)

${ }^{52}$ A. M. Ferrenberg and R. H. Swendsen, Phys. Rev. Lett. 63, 1195 (1989).

${ }^{53}$ A. Bellemans, J. Orban, and D. V. Belle, Mol. Phys. 39, 781 (1980).

${ }^{54}$ D. C. Rapaport, J. Phys. A 11, L213 (1978).

${ }^{55}$ D. C. Rapaport, J. Chem. Phys. 71, 3299 (1979).

${ }^{56}$ A. E. Sherwood and J. M. Prausnitz, J. Chem. Phys. 41, 429 (1964).

${ }^{57}$ C. P. Bokis, M. D. Donohue, and C. K. Hall, Ind. Eng. Chem. Res. 33, 1290 (1994).

${ }^{58}$ M. Banaszak and Y. C. Chiew, Phys. Rev. E 48, 3760 (1993).

${ }^{59}$ Y. Zhou, C. K. Hall, and G. Stell, Mol. Phys. 86, 1485 (1995).

${ }^{60}$ R. J. Baxter, J. Chem. Phys. 49, 2770 (1968).

${ }^{61}$ J. K. Lee, J. A. Barker, and F. F. Abraham, J. Chem. Phys. 58, 3166 (1973).

${ }^{62}$ R. Dickman and C. K. Hall, J. Chem. Phys. 85, 4108 (1986).

${ }^{63}$ N. Metropolis, A. W. Rosenbluth, M. N. Rosenbluth, A. H. Teller, and E. Teller, J. Chem. Phys. 21, 1087 (1953).

${ }^{64}$ H. L. Friedman, A Course in Statistical Mechanics (Prentice-Hall, Englewood Cliffs, 1985).

${ }^{65}$ M. P. Allen and D. J. Tildesley, Computer Simulation of Liquids (Oxford University Press, Oxford, 1987).

${ }^{66}$ L. V. Woodcock, Chem. Phys. Lett. 10, 257 (1971).

${ }^{67}$ H. C. Andersen, J. Chem. Phys. 72, 2384 (1980).

${ }^{68}$ W. G. Hoover, Annu. Rev. Phys. Chem. 34, 103 (1983).

${ }^{69}$ S. Nose, Mol. Phys. 52, 255 (1984).

${ }^{70}$ M. Krajci, Comput. Phys. Commun. 42, 29 (1986).

${ }^{71}$ M. E. Clamp, P. G. Baker, C. J. Stirling, and A. Brass, J. Comput. Chem. 15, 838 (1994).

${ }^{72}$ B. M. Forrest and U. W. Suter, J. Chem. Phys. 101, 2616 (1994).

${ }^{73}$ D. G. Gromov and J. J. de Pablo, J. Chem. Phys. 103, 8247 (1995).

${ }^{74}$ R. D. Present, Kinetic Theory of Gases (McGraw-Hill, New York, 1958), p. 32.

${ }^{75}$ D. J. Evans and G. P. Morriss, Comput. Phys. Rep. 1, 297 (1984).

${ }^{76}$ J. M. Haile and S. Gupta, J. Chem. Phys. 79, 3067 (1983).

${ }^{77}$ L. Verlet, Phys. Rev. 159, 98 (1967). 
${ }^{78}$ B. Quentrec and C. Brot, J. Comput. Phys. 13, 430 (1975).

${ }^{79}$ J. J. Erpenbeck and W. W. Wood, in Modern Theoretical Chemistry, edited by B. J. Berne (Plenum, New York, 1977).

${ }^{80}$ A. M. Ferrenberg and R. H. Swendsen, Phys. Rev. Lett. 61, 2635 (1988).

${ }^{81}$ F. A. Lindemann, Phys. Z. 11, 609 (1910).

${ }^{82}$ L. Cartz, Proc. Phys. Soc. London, Sect. B 68, 957 (1955).

${ }^{83}$ J. H. Bilgram, Phys. Rep. 153, 1 (1987).

${ }^{84}$ H. Löwen, Phys. Rep. 237, 249 (1994).

${ }^{85}$ F. H. Stillinger, Science 267, 1935 (1995).

${ }^{86}$ F. H. Stillinger and D. K. Stillinger, J. Chem. Phys. 93, 6013 (1990).

${ }^{87}$ W. Kabsch, Acta Crystallogr. A 32, 922 (1976).

${ }^{88}$ P. G. de Gennes, Scaling Concepts in Polymer Physics (Cornell University Press, Ithaca, 1979).

${ }^{89}$ J. Wichert and C. K. Hall, Macromolecules 27, 2744 (1994).

${ }^{90}$ E. Leontidis, J. J. de Pablo, M. Laso, and U. W. Suter, Adv. Polym. Sci. 116, 283 (1994).

${ }^{91}$ R. H. Boyd, Macromolecules 22, 2477 (1989).

${ }^{92}$ N. G. Almarza, E. Enciso, and F. J. Bermejo, J. Chem. Phys. 96, 4625 (1992).

${ }^{93}$ J. I. Siepmann, Mol. Phys. 70, 1145 (1990).

${ }^{94}$ J. I. Siepmann and D. Frenkel, Mol. Phys. 75, 59 (1992).

${ }^{95}$ J. J. de Pablo, M. Laso, and U. W. Suter, J. Chem. Phys. 96, 2395 (1992).

${ }^{96}$ L. Dodd, T. D. Boone, and D. Theodorou, Mol. Phys. 78, 961 (1993).

${ }^{97}$ L. Dodd and D. Theodorou, Adv. Polym. Sci. 116, 249 (1994).

${ }^{98}$ Y. Zhou and M. Karplus, Mol. Phys. 89, 1707 (1996).

${ }^{99}$ A. Baumgärtner, J. Chem. Phys. 72, 871 (1980).

${ }^{100}$ A. Baumgärtner, J. Chem. Phys. 73, 2489 (1980).

${ }^{101}$ For a finite system, a "discontinuity" reflects a smooth transition in a very narrow temperature range.

${ }^{102}$ D. A. Young and B. J. Alder, J. Chem. Phys. 60, 1254 (1974).

${ }^{103}$ M. J. Buerger, in Phase Transformations in Solids, edited by R. Smoluchowski, J. E. Meyer, and W. A. Weyl (Wiley, New York, 1951).

${ }^{104}$ F. L. McCrackin, J. Mazur, and C. M. Guttman, Macromolecules 6, 859 (1973).
${ }^{105}$ K. Kremer, A. Baümgartner, and K. Binder, J. Phys. A 15, 2879 (1981).

${ }^{106}$ C. M. Guttman, F. L. McCrackin, and C. C. Han, Macromolecules 15, 1205 (1982).

${ }^{107}$ W. Bruns, Macromolecules 17, 2826 (1984).

${ }^{108}$ W. Bruns and W. Carl, Macromolecules 24, 209 (1991).

${ }^{109}$ A. Kolinski, J. Skolnick, and R. Yaris, J. Chem. Phys. 85, 3585 (1986).

${ }^{110}$ A. Kolinski, J. Skolnick, and R. Yaris, Macromolecules 20, 438 (1987).

${ }^{111}$ I. Naghizadeh and J. Kovac, Phys. Rev. Lett. 59, 1710 (1987).

${ }^{112}$ J. P. Downey and J. Kovac, Macromolecules 23, 3013 (1990).

${ }^{113}$ H. Meirovitch and H. A. Lim, J. Chem. Phys. 92, 5144 (1990).

${ }^{114}$ C. C. Sorensen and J. Kovac, Macromolecules 24, 3883 (1991).

${ }^{115}$ X. Yuan and A. J. Masters, J. Chem. Phys. 94, 6908 (1991).

${ }^{116}$ I. Webman, J. L. Lebowitz, and M. H. Kalos, Macromolecules 14, 1495 (1981).

${ }^{117}$ M. Bishop and J. Michels, J. Chem. Phys. 84, 447 (1986).

${ }^{118}$ J. J. Freire, J. Pla, A. Rey, and R. Prats, Macromolecules 19, 452 (1986).

${ }^{119}$ A. Rey, J. J. Freire, and J. G. de-la Torre, Macromolecules 20, 2385 (1987).

${ }^{120}$ N. Karasawa and W. A. Goddard III, J. Phys. Chem. 92, 5828 (1988).

${ }^{121}$ P. Bladon and D. Frenkel, Phys. Rev. Lett. 74, 2519 (1995).

${ }^{122}$ A. H. Marcus and S. A. Rice, Phys. Rev. Lett. 77, 2577 (1996).

${ }^{123}$ Y. Zhou and M. Karplus, Proc. Natl. Acad. Sci. USA (in press).

${ }^{124}$ L. Vega, E. de Miguel, L. F. Rull, G. Jackson, and L. A. McLure, J. Chem. Phys. 96, 2296 (1992).

${ }^{125}$ B. R. Brooks, R. E. Bruccoleri, B. D. Olafson, D. J. States, S. Swaminathan, and M. Karplus, J. Comput. Chem. 4, 187 (1983).

${ }^{126}$ E. Neria, S. Fischer, and M. Karplus, J. Chem. Phys. 105, 1902 (1996).

${ }^{127}$ O. B. Ptitsyn, Adv. Protein Chem. 47, 83 (1995).

${ }^{128}$ N. Go, Int. J. Peptide Protein Res. 7, 313 (1975).

${ }^{129}$ J. C. Nelson, J. G. Saven, J. S. Moore, and P. G. Wolynes, Science 277, 1793 (1997). 